\title{
Phenomenological implications of deflected mirage mediation: comparison with mirage mediation
}

\author{
Baris Altunkaynak, ${ }^{a}$ Brent D. Nelson, ${ }^{a}$ Lisa L. Everett, ${ }^{b}$ lan-Woo $\mathrm{Kim}^{b}$ and \\ Yongyan $\mathbf{R a o}^{b}$ \\ a Department of Physics, Northeastern University, \\ 110 Forsyth St, 111 Dana Research Center Boston, MA 02115, U.S.A. \\ ${ }^{b}$ Department of Physics, University of Wisconsin-Madison, \\ 1150 University Avenue, Madison, WI 53706, U.S.A. \\ E-mail: baris.altunkaynak@gmail.com, b.nelson@neu.edu, \\ leverett@wisc.edu, ikim@physics.wisc.edu, yrao2@wisc.edu
}

ABSTRACT: We compare the collider phenomenology of mirage mediation and deflected mirage mediation, which are two recently proposed "mixed" supersymmetry breaking scenarios motivated from string compactifications. The scenarios differ in that deflected mirage mediation includes contributions from gauge mediation in addition to the contributions from gravity mediation and anomaly mediation also present in mirage mediation. The threshold effects from gauge mediation can drastically alter the low energy spectrum from that of pure mirage mediation models, resulting in some cases in a squeezed gaugino spectrum and a gluino that is much lighter than other colored superpartners. We provide several benchmark deflected mirage mediation models and construct model lines as a function of the gauge mediation contributions, and discuss their discovery potential at the LHC.

KEYwORDS: Supersymmetry Phenomenology

ArXiv EPRINT: 1001.5261 


\section{Contents}

1 Introduction $\quad 1$

2 Theoretical framework 3

3 (Deflected) mirage unification and particle mass spectra $\quad 7$

$\begin{array}{lll}3.1 & \text { General features of deflected mirage mediation models } & 7\end{array}$

3.2 Mirage mediation and deflected mirage mediation model pairs 11

4 Collider phenomenology $\quad \mathbf{1 5}$

4.1 Phenomenology of mirage and deflected mirage mediation model pairs $\quad 15$

$\begin{array}{ll}4.2 & \text { Influence of } \alpha_{g} \text { on LHC phenomenology } \\ \end{array}$

5 Conclusions $\quad 30$

$\begin{array}{ll}\text { A Anomalous dimensions } & 31\end{array}$

\section{Introduction}

TeV scale softly broken supersymmetry (SUSY) (for recent reviews, see [1, 2]) is one of the best-motivated candidates for physics beyond the Standard Model (SM). Theories with softly broken supersymmetry, such as the minimal supersymmetric standard model (MSSM), will be tested thoroughly at the Large Hadron Collider (LHC). The collider phenomenology of such theories depends in detail on the associated soft supersymmetry breaking terms, which in turn are largely governed by the way in which supersymmetry breaking is mediated from a hidden sector to the SM fields. As the number of possible soft supersymmetry breaking parameters is vast even in minimal extensions of the SM such as the MSSM, it is fruitful to develop models of supersymmetry breaking. Exploring the possible mediation mechanisms for supersymmetry breaking is crucially important for LHC tests of the hypothesis that supersymmetry is present at TeV energy scales.

Viable supersymmetry breaking models are based on the hidden sector paradigm, in which supersymmetry is broken in a hidden or secluded sector and communicated to the observable sector via the interactions of mediator fields. As is well known, the phenomenological implications of these models are largely insensitive to the details of the hidden sector, and instead are governed by the mediation mechanism that is responsible for the transmission of supersymmetry breaking to the MSSM fields. Most models of supersymmetry breaking involve one of the three most popular mediation mechanisms: (i) gravity mediation, (ii) gauge mediation, and (iii) (braneworld-motivated) "bulk" mediation models. Gravity-mediated terms [3-7] arise from couplings that vanish as the Planck mass $M_{P} \rightarrow \infty$; examples include the (tree-level) minimal supergravity (mSUGRA) model 
(also known as the constrained MSSM (CMSSM)) and modulus mediation models [8-10], and the (loop-suppressed) anomaly mediation models [11-13]. Gauge-mediated terms arise from loop diagrams involving new messenger fields with SM charges [14-23]. Bulk-mediated terms arise from bulk mediator fields in braneworld scenarios. Examples include gaugino mediation [24] and $Z^{\prime}$ mediation [26, 27]. Certain gravity-mediated models, such as the pure anomaly mediation scenario [11-13] (which requires sequestering), are also bulk mediation models. Typically, one of these mediation mechanisms is assumed to dominate (see e.g. [28]), for simplicity and practicality and/or to solve a given problem of the MSSM, such as the $\mu$ problem or the flavor/CP problems.

A complementary approach is to consider models in which more than one mediation mechanism plays an important role. Such "mixed" scenarios are motivated within stringmotivated constructions, such as the Kachru-Kallosh-Linde-Trivedi (KKLT) approach to moduli stabilization [29]. A prototype KKLT-motivated example is mirage mediation [30, 31], a phenomenological model in which the tree-level gravity (modulus)-mediated terms and the (loop-suppressed) anomaly-mediated terms are comparable in size, contrary to naive expectations. As a result, the soft masses unify at a scale that is typically well below the scale where they are generated, resulting in "mirage" unification. Mirage mediation has distinctive phenomenological implications compared with standard minimal supergravity models [32-38]. These features include a gaugino mass pattern that is typically more squeezed than the standard mSUGRA/CMSSM gaugino mass pattern [39] and reduced low energy fine-tuning [40-44], for which the details depend on the ratio of the gravitymediated contributions to the anomaly-mediated contributions.

The recently proposed deflected mirage mediation scenario is an extension of mirage mediation in which gauge-mediated supersymmetry breaking terms are also present and competitive in size to the gravity-mediated and anomaly-mediated soft terms [45, 46]. This framework is denoted as deflected mirage mediation because the mirage unification scale in the gaugino sector is shifted or "deflected" from its mirage mediation value due to threshold effects associated with the gauge mediation messengers fields. In deflected mirage mediation, the gauge-mediated terms arise from the couplings of an additional matter modulus field $X$ and vectorlike messenger pairs with nontrivial SM quantum numbers. The ratio of the gauge-mediated and anomaly-mediated terms depends on the details of the stabilization mechanism for the mediator field $X$. In [46], it was found that if the stabilization mechanism is dominated by supersymmetry breaking effects, generically the gauge-mediated and anomaly-mediated contributions are comparable. Deflected mirage mediation provides a general framework in which to explore mixed supersymmetry breaking scenarios at the LHC, where well-known single mediation mechanism models can be recovered by judiciously adjusting dimensionless parameters in the theory.

In this paper, we explore the question of how the collider phenomenology of deflected mirage mediation differs from that of standard mirage mediation, following previous work on the sparticle spectrum [46, 47] and dark matter constraints [48]. More precisely, we focus our attention on the effects of the gauge mediated terms by varying the ratio of gauge to anomaly mediation contributions and/or the number of messenger pairs, as compared to benchmark KKLT mirage mediation scenarios. The differences in the collider 
phenomenology of the deflected mirage mediation scenarios compared to the corresponding pure mirage mediation scenarios depend primarily on the deflection of the gaugino mass mirage unification scale. This depends in turn on the size of the messenger scale and whether the threshold effects are large or small. For small threshold effects, the pattern of soft masses does not differ greatly from the corresponding pure mirage mediation limit. However, if the threshold effects are large, one can have situations in which the gaugino mirage unification scale can be deflected from a high scale value to the $\mathrm{TeV}$ scale. In such situations, the gaugino mass spectrum is squeezed, resulting in gluinos that are typically lighter than other colored superpartners. The phenomenology of light gluino scenarios has been studied for example in [49-55]. Unlike the case of the constrained MSSM, the LHC phenomenology in such cases is dominated by gluino production, with soft decay products due to the compressed chargino and neutralino mass spectrum.

The outline of this paper is as follows. In section 2, we review the theoretical framework of deflected mirage mediation and enumerate the supersymmetry breaking terms of the theory. An overview of mirage unification and the properties of the resulting low energy mass spectra in both mirage mediation and deflected mirage mediation is given in section 3 . We then discuss the collider phenomenology of benchmark deflected mirage mediation models and compare it to that of pure mirage mediation benchmark scenarios in section 4 . In section 5, we provide our conclusions and outlook.

\section{Theoretical framework}

We begin with a brief review of mirage mediation, a phenomenological model motivated from the KKLT flux compactification approach within Type IIB string theory [29]. In this setup, the MSSM fields are confined to a stack of D branes that are localized in a higher-dimensional bulk Calabi-Yau space, and the hidden (supersymmetry breaking) sector consists of anti-branes at the tip of the warped throat geometry. The tree-level gravity mediation terms are

$$
m_{\text {soft }}^{\text {(modulus) }} \sim \frac{F^{T}}{T+\bar{T}}
$$

where $T$ is the Kähler modulus. The anomaly mediation terms are

$$
m_{\text {soft }}^{(\text {anomaly })} \sim \frac{1}{16 \pi^{2}} \frac{F^{C}}{C}
$$

where $C$ is the conformal compensator of the gravity multiplet. In the KKLT scenario, there is a cancellation between the superpotential terms from the fluxes and the nonperturbative terms, leading to a supersymmetry-preserving vacuum with stabilized moduli but with a negative cosmological constant. Supersymmetry is then broken by an uplifting potential of the form $(T+\bar{T})^{-n_{p}}$, where $n_{p}=2$ in the KKLT model, due to the anti-branes at the tip of the warped throat. After cancelling the cosmological constant, the following mirage mediation relation is obtained [30, 31]:

$$
\frac{F^{T}}{T+\bar{T}} \sim \frac{1}{\ln \left(M_{P} / m_{3 / 2}\right)} \frac{F^{C}}{C},
$$


in which $M_{P}=2.4 \times 10^{18} \mathrm{GeV}$ is the reduced Planck mass, and $m_{3 / 2}$ is the gravitino mass. As $m_{3 / 2}$ is typically $\sim 100 \mathrm{TeV}$ in this class of models, the factor of $\ln \left(M_{P} / m_{3 / 2}\right)$ is numerically close to $4 \pi^{2}$. Hence, the tree-level gravity mediation terms are comparable to the anomaly mediation terms in mirage mediation.

In deflected mirage mediation, the observable sector matter content also includes a gauge singlet $X$ and vectorlike messenger pairs $\Psi, \bar{\Psi}$ with SM gauge charges, which are fields that are generically present in string-derived models. In general, $X$ can acquire an $F$ term vacuum expectation value, leading to gauge mediated terms of the form

$$
m_{\text {soft }}^{\text {(gauge) }} \sim \frac{1}{16 \pi^{2}} \frac{F^{X}}{X} .
$$

Depending on the stabilization mechanism for $X$, it was shown in $[45,46]$ that in general,

$$
\frac{F^{X}}{X} \sim \frac{F^{C}}{C}
$$

such that $m_{\mathrm{soft}}^{\text {(gauge) }}$ is comparable to $m_{\mathrm{soft}}^{\text {(anom) }}$ and $m_{\mathrm{soft}}^{\text {(grav) }}$ in deflected mirage mediation.

To see this more clearly, let us begin with the effective supergravity theory of KKLTinspired models. Labeling the observable sector (MSSM) fields as $\Phi$ and taking a diagonal matter metric for simplicity, the Kähler potential at leading order is

$$
K=-3 \log (T+\bar{T})+\frac{X \bar{X}}{(T+\bar{T})^{n_{X}}}+\frac{\Phi_{i} \bar{\Phi}_{i}}{(T+\bar{T})^{n_{i}}},
$$

where $n_{X}$ and $n_{i}$ are the modular weights of $X$ and $\Phi_{i}$, respectively. The superpotential is

$$
W=W_{0}+W_{1}(X)+\lambda X \Psi \bar{\Psi}+W_{\mathrm{MSSM}}
$$

In eq. (2.7), $W_{0}$ is the part of the superpotential that governs (together with the uplifting potential) the supersymmetry breaking effects [29], $W_{1}(X)=\lambda_{n} X^{n}$ describes the possible self-couplings of $X$, and $W_{\text {MSSM }}$ takes the form

$$
W_{\mathrm{MSSM}}=\mu_{i j}^{0} \Phi_{i} \Phi_{j}+y_{i j k}^{0} \Phi_{i} \Phi_{j} \Phi_{k}
$$

in which $\mu_{i j}^{0}$ are supersymmetric mass parameters, and $y_{i j k}^{0}$ are the (unnormalized) Yukawa couplings. The gauge kinetic functions are assumed to take the form

$$
f_{a}\left(M_{\mathrm{G}}\right)=T .
$$

The messengers are taken to be $\Psi, \bar{\Psi}$ are $5, \overline{5}$ representations of $\mathrm{SU}(5)$, as is standard in many models of gauge mediation. Here $N$ will denote the number of such messenger pairs.

Upon computing the soft terms using standard supergravity techniques, we obtain the observable sector soft supersymmetry breaking Lagrangian, which is of the usual form

$$
\mathcal{L}_{\text {soft }}=-m_{i}^{2}\left|\Phi^{i}\right|^{2}-\left[\frac{1}{2} M_{a} \lambda^{a} \lambda^{a}+A_{i j k} y_{i j k} \Phi^{i} \Phi^{j} \Phi^{k}+\text { h.c. }\right]
$$


in which $m_{i}^{2}$ are the soft scalar mass-squared parameters, $M_{a}$ are the gaugino masses, and $A_{i j k}$ are trilinear scalar interaction parameters. ${ }^{1}$

Recalling that above the mass scale of the messengers $M_{\text {mess }} \equiv \lambda\langle X\rangle$, the beta functions depend on not only the MSSM fields, but also on the messenger pairs, the soft terms at the GUT scale $M_{\mathrm{G}}$ and the messenger threshold effects at $M_{\text {mess }}$ are as follows:

Gaugino masses. The gaugino mass parameters are given by

$$
\begin{aligned}
M_{a}\left(M_{\mathrm{G}}\right) & =\frac{F^{T}}{T+\bar{T}}+\frac{g_{0}^{2}}{16 \pi^{2}} b_{a}^{\prime} \frac{F^{C}}{C} \\
M_{a}\left(M_{\text {mess }}^{-}\right) & =M_{a}\left(M_{\text {mess }}^{+}\right)+\Delta M_{a},
\end{aligned}
$$

in which the threshold corrections are

$$
\Delta M_{a}=-N \frac{g_{a}^{2}\left(M_{\mathrm{mess}}\right)}{16 \pi^{2}}\left(\frac{F^{C}}{C}+\frac{F^{X}}{X}\right) .
$$

Here $g_{0}$ is the unified gauge coupling at $M_{\mathrm{G}}$, and the beta functions $b_{a}^{\prime}$ are related to their MSSM counterparts by $b_{a}^{\prime}=b_{a}+N$, with $\left(b_{3}, b_{2}, b_{1}\right)=\left(-3,1, \frac{33}{5}\right)$ (in our conventions, $b_{a}<0$ for asymptotically free theories).

Trilinear terms. The trilinear terms are $A_{i j k}=A_{i}+A_{j}+A_{k}$, with

$$
A_{i}\left(\mu=M_{\mathrm{G}}\right)=\left(1-n_{i}\right) \frac{F^{T}}{T+\bar{T}}-\frac{\gamma_{i}}{16 \pi^{2}} \frac{F^{C}}{C},
$$

where $\gamma_{i}$ is the anomalous dimension of $\Phi_{i}$.

Soft scalar masses. The scalar mass-squared parameters are given by

$$
\begin{aligned}
m_{i}^{2}\left(\mu=M_{\mathrm{G}}\right) & =\left(1-n_{i}\right)\left|\frac{F^{T}}{T+\bar{T}}\right|^{2}-\frac{\theta_{i}^{\prime}}{32 \pi^{2}}\left(\frac{F^{T}}{T+\bar{T}} \frac{F^{\bar{C}}}{\bar{C}}+\text { h.c. }\right)-\frac{\dot{\gamma}_{i}^{\prime}}{\left(16 \pi^{2}\right)^{2}}\left|\frac{F^{C}}{C}\right|^{2}, \\
m_{i}^{2}\left(\mu=M_{\text {mess }}^{-}\right) & =m_{i}^{2}\left(\mu=M_{\text {mess }}^{+}\right)+\Delta m_{i}^{2},
\end{aligned}
$$

where the threshold corrections are

$$
\Delta m_{i}^{2}=\sum_{a} 2 c_{a} N \frac{g_{a}^{4}\left(M_{\text {mess }}\right)}{\left(16 \pi^{2}\right)^{2}}\left(\left|\frac{F^{X}}{X}\right|^{2}+\left|\frac{F^{C}}{C}\right|^{2}+\frac{F^{X}}{X} \frac{F^{\bar{C}}}{\bar{C}}+\text { h.c. }\right) .
$$

In the above, $c_{a}$ is the quadratic Casimir, and $\gamma_{i}, \dot{\gamma}_{i}, \theta_{i}\left(\gamma_{i}^{\prime}, \dot{\gamma}_{i}^{\prime}, \theta_{i}^{\prime}\right)$ are listed in appendix A. We now replace the $\mathrm{F}$ terms with the parameterization given in $[45,46]$, as follows:

$$
\begin{aligned}
& \frac{F^{C}}{C}=\alpha_{m} \ln \frac{M_{P}}{m_{3 / 2}} \frac{F^{T}}{T+\bar{T}}=\alpha_{m} \ln \frac{M_{P}}{m_{3 / 2}} M_{0} \\
& \frac{F^{X}}{X}=\alpha_{g} \frac{F^{C}}{C}=\alpha_{g} \alpha_{m} \ln \frac{M_{P}}{m_{3 / 2}} M_{0},
\end{aligned}
$$

\footnotetext{
${ }^{1}$ These terms are defined in the field basis in which the kinetic terms are canonically normalized; the physical Yukawa couplings $y_{i j k}=y_{i j k}^{0} /\left(Z_{i} Z_{j} Z_{k}\right)^{1 / 2}$ are in the definition of trilinear terms.
} 
in which $M_{0} \equiv F^{T} /(T+\bar{T})$ sets the overall scale of the soft terms. The dimensionless parameter $\alpha_{m}$ is the $\alpha$ parameter of mirage mediation: it denotes the relative importance of anomaly mediation with respect to gravity mediation. In the KKLT case $\alpha_{m}=1$; the case of $\alpha_{m}=2$ (i.e, an uplifting potential with $n_{p}=1$, which has no obvious string theory realization) has also been considered in the literature, for reasons that will be clear shortly.

The dimensionless parameter $\alpha_{g}$ denotes the relative importance of the gauge-mediated terms with respect to the anomaly-mediated terms. For positive values of $\alpha_{g}$, the threshold corrections to the soft terms from gauge mediation add constructively to the threshold effects from anomaly mediation, while for negative values of $\alpha_{g}$ the two contributions destructively interfere. We will see later in the paper that the sign of $\alpha_{g}$ thus has important consequences for the TeV scale phenomenology of these models. The values of $\alpha_{g}$ depend on the details of the stabilization of $X[45,46]$. For radiative stabilization,

$$
\frac{F^{X}}{X}=-\frac{F^{C}}{C}
$$

while for self-couplings of the form $W_{1}=\lambda_{n} X^{n}$,

$$
\frac{F^{X}}{X}=-\frac{2}{n-1} \frac{F^{C}}{C} \text {. }
$$

Here $n$ is restricted to the values $n \geq 3$ or $n<0$, i.e., stabilization by higher-dimensional operators or non-perturbative effects, respectively. We see that higher-dimensional operators result in models with $\alpha_{g}<0$, and non-perturbative effects lead to $\alpha_{g}>0$. With the parametrization given in eqs. (2.17)-(2.18), the soft terms at $M_{G}$ take the form

$$
\begin{aligned}
& M_{a}\left(M_{\mathrm{G}}\right)=M_{0}\left[1+\frac{g_{0}^{2}}{16 \pi^{2}} b_{a}^{\prime} \alpha_{m} \ln \frac{M_{P}}{m_{3 / 2}}\right] \\
& A_{i}\left(M_{\mathrm{G}}\right)=M_{0}\left[\left(1-n_{i}\right)-\frac{\gamma_{i}}{16 \pi^{2}} \alpha_{m} \ln \frac{M_{P}}{m_{3 / 2}}\right] \\
& m_{i}^{2}\left(M_{\mathrm{G}}\right)=M_{0}^{2}\left[\left(1-n_{i}\right)-\frac{\theta_{i}^{\prime}}{16 \pi^{2}} \alpha_{m} \ln \frac{M_{P}}{m_{3 / 2}}-\frac{\dot{\gamma}_{i}^{\prime}}{\left(16 \pi^{2}\right)^{2}}\left(\alpha_{m} \ln \frac{M_{P}}{m_{3 / 2}}\right)^{2}\right],
\end{aligned}
$$

and the threshold terms are given by

$$
\begin{aligned}
& \Delta M_{a}=-M_{0} N \frac{g_{a}^{2}\left(M_{\text {mess }}\right)}{16 \pi^{2}} \alpha_{m}\left(1+\alpha_{g}\right) \ln \frac{M_{P}}{m_{3 / 2}}, \\
& \Delta m_{i}^{2}=M_{0}^{2} \sum_{a} 2 c_{a} N \frac{g_{a}^{4}\left(M_{\text {mess }}\right)}{\left(16 \pi^{2}\right)^{2}}\left[\alpha_{m}\left(1+\alpha_{g}\right) \ln \frac{M_{P}}{m_{3 / 2}}\right]^{2} .
\end{aligned}
$$

The parameters of the model are the mass scales $M_{0}$ and $M_{\text {mess }}$, as well as the dimensionless quantities $\alpha_{m}, \alpha_{g}$, the number of $\mathrm{SU}(5)$ messenger pairs $N$, the modular weights $n_{i}, \tan \beta$, and $\operatorname{sign} \mu$ (the model-dependent $\mu$ and $B \mu$ parameters are replaced as usual by the $Z$ boson mass, $\tan \beta$, and the sign of $\mu$ ). Here, we will fix the modular weights to the standard values $n_{i}=1 / 2$ for all SM matter fields, and $n_{i}=1$ for the MSSM Higgs doublets. With this choice, we see that in deflected mirage mediation, in addition to the usual $\tan \beta$ and $\operatorname{sign} \mu$, 
there are the continuous parameters $M_{0}$ and $M_{\text {mess }}$, the discrete parameter $N$, and the parameters $\alpha_{m}$ and $\alpha_{g}$, which take on discrete values within a particular string framework but can be taken to be continuous parameters within a purely phenomenological approach.

Hence, deflected mirage mediation models in this class have six parameters plus one sign. This is to be compared with the four continous parameters (the universal scalar mass $M_{0}$, the universal gaugino mass $M_{1 / 2}$, and the universal trilinear scalar coupling $A_{0}$, and $\tan \beta$ ) plus one sign (the sign of $\mu$ ) in mSUGRA/CMSSM models. We will see that despite its relatively small extension of parameters compared to mSUGRA/CMSSM models, deflected mirage mediation provides a rich framework for LHC phenomenology.

\section{3 (Deflected) mirage unification and particle mass spectra}

\subsection{General features of deflected mirage mediation models}

To compare deflected mirage mediation to mirage mediation, we provide here a discussion of the prototypical feature of mirage mediation, which is the phenomenon of mirage unification and its resulting profound impact on the low energy spectrum (see also [45-47] for previous discussions). Mirage mediation is thus named because at the one-loop order, the soft terms unify not at the unification scale $M_{G} \sim 10^{16} \mathrm{GeV}$ as in the case of mSUGRA/CMSSM models, but rather at a "mirage" scale [30-32]:

$$
M_{\text {mir }}=M_{G}\left(\frac{m_{3 / 2}}{M_{P}}\right)^{\alpha_{m} / 2},
$$

where $\alpha_{m}$ is the ratio of anomaly mediation to gravity mediation terms given in eq. (2.17). For the KKLT value of $\alpha_{m}=1$, the mirage unification occurs at $\sim 10^{10} \mathrm{GeV}$, as shown in figure 1. Here we have included two-loop effects in the running, which spoil the precise unification, though the general features are maintained. The mass spectrum, also given in figure 1, shows that this model has a relatively heavy gluino, but has a slightly compressed spectrum with respect to corresponding mSUGRA/CMSSM models. Smaller values of $\alpha_{m}$ have mirage unification scales closer to $M_{G}$ (with $M_{\text {mir }}=M_{G}$ in the limit of $\alpha_{m}=0$ ), while larger values of $\alpha_{m}$ result in lower mirage unification scales. The case of $\alpha_{m}=2$ results in a mirage unification scale at $\mathrm{TeV}$ energies, resulting in a highly compressed superpartner spectrum and a reduced little hierarchy problem [33-36, 40-44].

In deflected mirage mediation, mirage unification is maintained in the gaugino sector (though lost in general in the scalar sector), as follows [45, 46]:

$$
M_{\mathrm{mir}}=M_{G}\left(\frac{m_{3 / 2}}{M_{P l}}\right)^{\alpha_{m} \rho / 2}
$$

with the parameter $\rho$ given by

$$
\rho=\left(1+\frac{2 N g_{0}^{2}}{16 \pi^{2}} \ln \frac{M_{\mathrm{GUT}}}{M_{\mathrm{mess}}}\right)\left(1-\frac{\alpha_{\mathrm{m}} \alpha_{\mathrm{g}} N g_{0}^{2}}{16 \pi^{2}} \ln \frac{M_{P}}{m_{3 / 2}}\right)^{-1} .
$$

Eqs. (3.2)-( 3.3) show that the mirage mediation limit is obtained for $N=0$ (i.e., the absence of messengers). If $\alpha_{g}=0$, corresponding to vanishing gauge mediation contributions, the messengers still contribute to anomaly mediation, as reflected in eq. (3.3). 

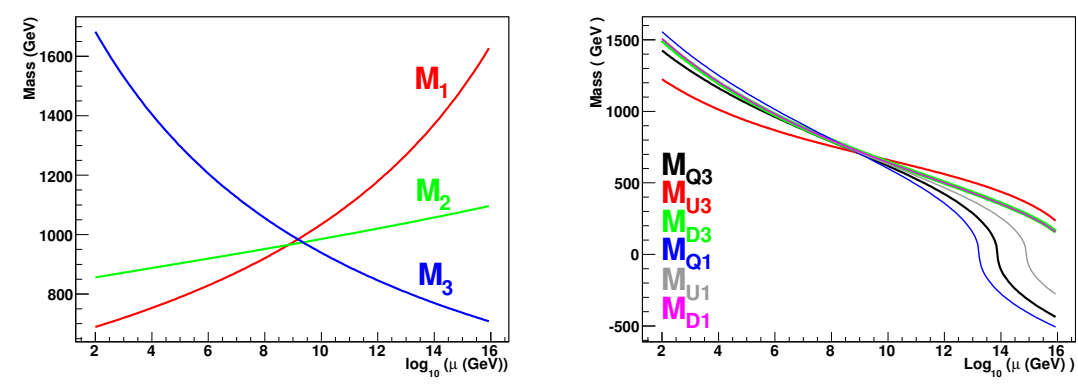

(a)

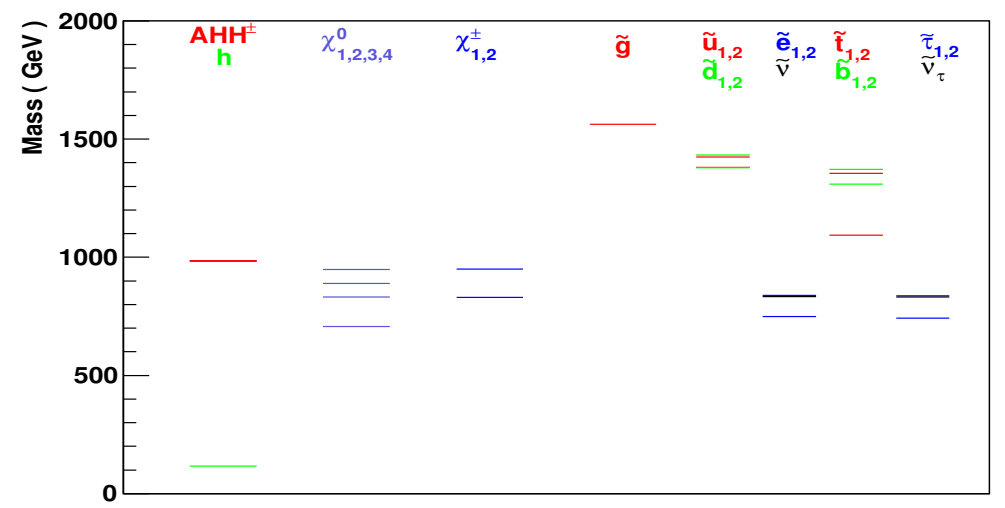

(b)

Figure 1. Mirage unification. The renormalization group evolution of the gauginos and soft mass-squared parameters (a) and the particle mass spectrum at low energies (b) for a pure mirage mediation scenario with $\alpha_{m}=1, M_{0}=1 \mathrm{TeV}, \tan \beta=10$, and $\mu>0$.

The gaugino mirage unification scale in deflected mirage mediation can change widely from the pure mirage mediation case with fixed $\alpha_{m}$, with the details depending on the values of $\alpha_{g}, N$, and the messenger scale $M_{\text {mess }}$. For nonzero $N$, the presence of the messengers results in $\rho>1$ when $\alpha_{g}=0$, such that the mirage unification scale is lowered compared to that of the pure mirage mediation case. For fixed $\alpha_{m}, N$, and $M_{\text {mess }}$, the effects of a nonzero $\alpha_{g}$ are straightforward to understand: for $\alpha_{g}>0, \rho$ increases and $M_{\text {mir }}$ is lowered, while for $\alpha_{g}<0, \rho$ decreases and $M_{\text {mir }}$ is correspondingly increased.

As an example of a model with gaugino mass mirage unification near the $\mathrm{TeV}$ scale, in figure 2 we show the renormalization group running of the gaugino and soft scalar masssquares, as well as the particle mass spectrum at the $\mathrm{TeV}$ scale, for the case of $\alpha_{g}=1$ and $M_{\text {mess }}=10^{12} \mathrm{GeV}$. Clearly, the gaugino mass spectrum is highly squeezed, with a very light gluino (the lightest of the colored superpartners). Indeed, for $N=3$ the beta-function coefficient for the soft supersymmetry breaking parameter $M_{3}$ vanishes at one loop above the messenger scale, and hence the soft mass for the gluino then runs very little between the high energy input scale and the messenger scale. Therefore, the gluino mass is much smaller for these cases than for analogous pure mirage mediation models. Furthermore, in contrast 

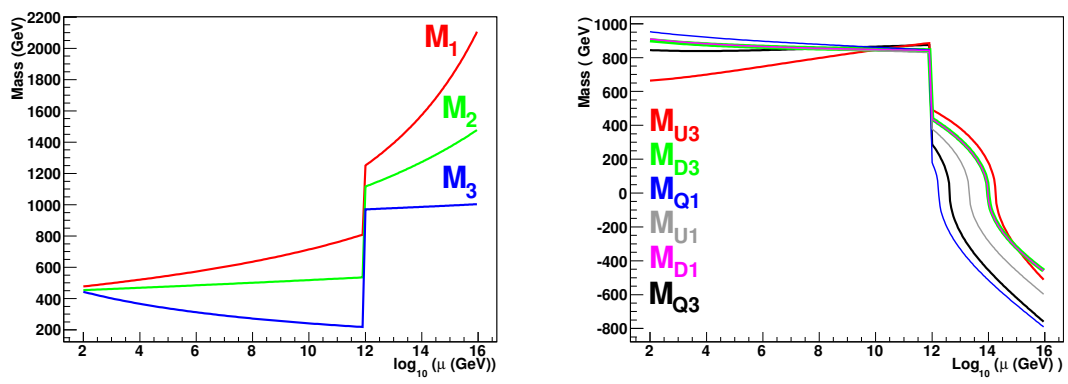

(a)

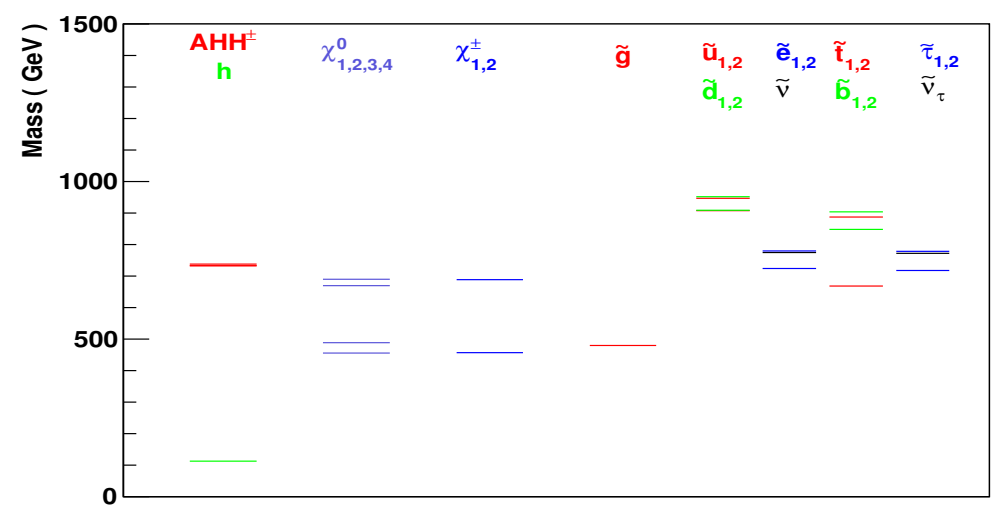

(b)

Figure 2. Deflected mirage unification. The renormalization group evolution of the gauginos and soft mass-squared parameters (a) and the mass spectrum (b) for a deflected mirage mediation model with $\alpha_{m}=1, \alpha_{g}=1, M_{\text {mess }}=10^{12} \mathrm{GeV}, N=3, M_{0}=1 \mathrm{TeV}, \tan \beta=10$, and $\mu>0$.

to the pure mirage mediation case with $\alpha_{m} \simeq 2$, which also has mirage unification near the $\mathrm{TeV}$ scale, the deflected mirage mediation scenarios with low-scale mirage unification have heavier scalars. The full superpartner spectrum is thus stretched with respect to the pure mediation scenario due to the effects of gauge mediation.

In contrast, we show in figure 3 a deflected mirage mediation scenario with the same parameters as that of the previous figure, except that $\alpha_{g}=-0.5$. In this case, there is a much higher gaugino mirage unification scale that is close to that of the pure mirage mediation limit, since with this choice of parameters $\rho \approx 1$. As a result, the particle mass spectrum strongly resembles that of figure 1, with heavier colored superpartners and no strong degeneracy between the lightest chargino and neutralino. This feature depends on the messenger scale; for $\alpha_{g}<0$, there are always pairs of $\alpha_{g}$ and $M_{\text {mess }}$ for which $\rho=1$ and thus $M_{\text {mir }}$ is given by the pure mirage mediation limit.

Furthermore, if the messenger scale is below the mirage unification scale as given in the pure mirage mediation case by eq. (3.1), the mirage unification behavior is maintained not only for the gauginos, but also for the scalars (though at a different scale than the pure mirage mediation limit due to the presence of the messengers). For $\alpha_{g}>0$, we can 

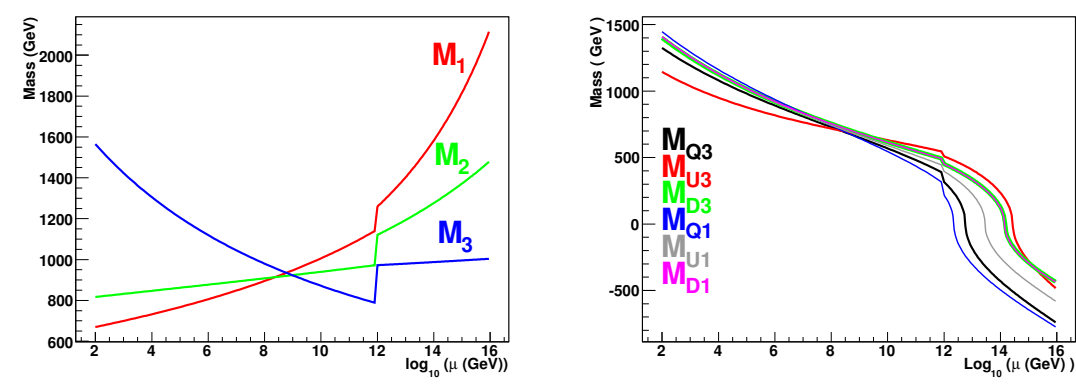

(a)

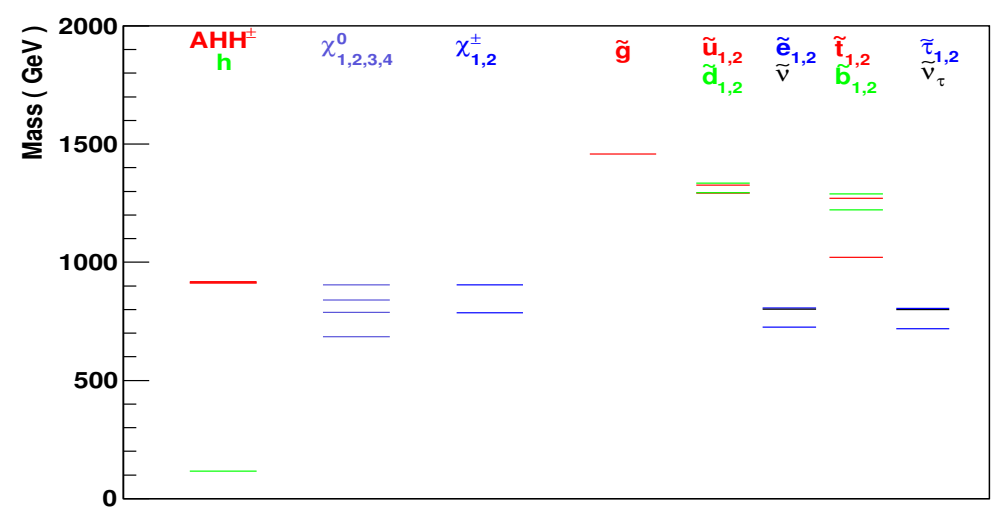

(b)

Figure 3. Deflected mirage unification. The renormalization group evolution of the gauginos and soft mass-squared parameters (a) and the mass spectrum (b) for a deflected mirage mediation model with $\alpha_{m}=1, \alpha_{g}=-0.5, M_{\text {mess }}=10^{12} \mathrm{GeV}, N=3, M_{0}=1 \mathrm{TeV}, \tan \beta=10$, and $\mu>0$.

find model points in which the gaugino mass unification scale is sub-TeV, resulting in a flipped gaugino mass spectrum in which the wino is the lightest superpartner, similar to the gaugino pattern in the pure anomaly mediation limit. An example is shown in figure 4, in which all parameters are the same as in figure 2, except that $M_{\text {mess }}=10^{5} \mathrm{GeV}$.

We see, therefore, that deflected mirage mediation models roughly can be categorized according to their values of $\alpha_{g}$ as follows: the case of large threshold effects $\left(\alpha_{g}>0\right.$, nonperturbative stabilization), and the case of small threshold effects $\left(\alpha_{g}<0\right.$, radiative or higher-dimensional stabilization). This can be easily understood from eqs. (2.21)-(2.25); for $\alpha_{g}>0, F^{X} / X$ and $F^{C} / C$ have the same sign, and thus the threshold effects for gauge and anomaly mediation constructively interfere, while for $\alpha_{g}<0$, the threshold effects destructively interfere. For small thresholds, the particle mass spectra are typically similar to a corresponding pure mirage mediation spectrum, while for large thresholds, deflected mirage mediation can result in significantly different mass spectra with non-standard gaugino mass patterns characterized by a squeezed gaugino mass spectrum with relatively light gluinos and a lightest superpartner with a mixed wino-bino-Higgsino content, similar to gauge messenger models $[56,57]$. This is phenomenologically interesting for a variety of 

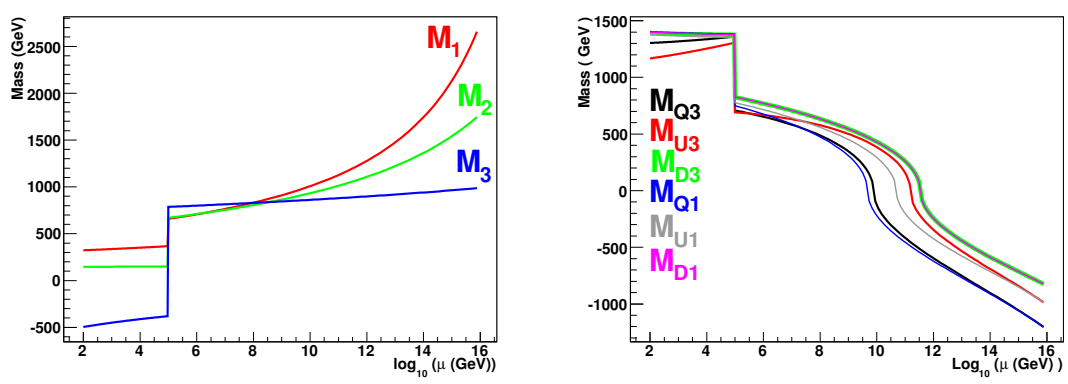

(a)

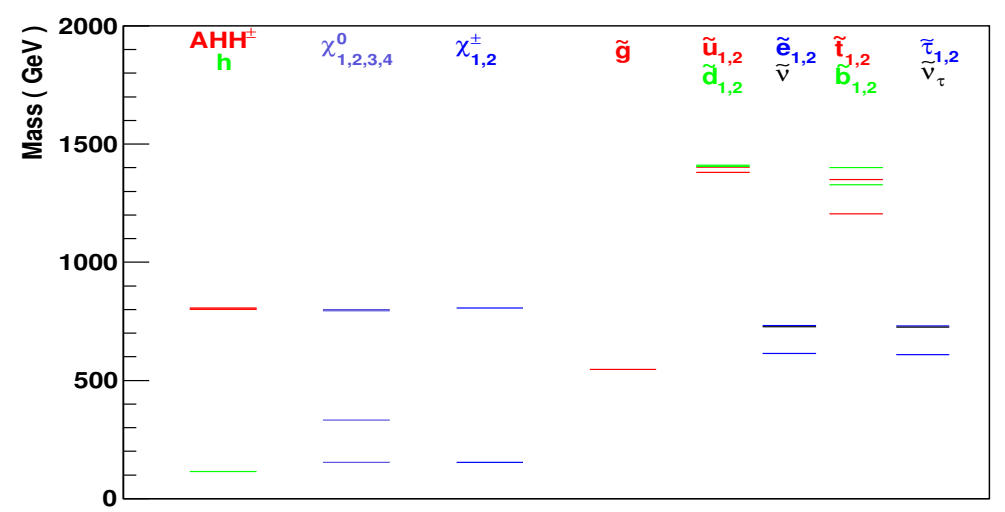

(b)

Figure 4. Deflected mirage unification. The renormalization group evolution of the gauginos and soft mass-squared parameters (a) and the mass spectrum (b) for a deflected mirage mediation scenario with $\alpha_{m}=1, \alpha_{g}=1, M_{\text {mess }}=10^{5} \mathrm{GeV}, N=3, M_{0}=1 \mathrm{TeV}, \tan \beta=10$, and $\mu>0$.

reasons, including fine-tuning considerations [40-44], LHC signatures [39, 58-60], and dark matter signals [48, 61-65]. However, the thresholds must not be too large, otherwise the gluino can become the lightest superpartner. Therefore, viable deflected mirage mediation models with large threshold effects have bounds on the allowed range of $\alpha_{g}$, depending on the other parameters. The examples we have shown here, while not chosen to optimize the dark matter predictions, encompass all of these possibilities.

\subsection{Mirage mediation and deflected mirage mediation model pairs}

As our goal in this paper is to identify the main differences for the $\mathrm{TeV}$ scale phenomenology of mirage mediation models and deflected mirage mediation models, we now propose a set of benchmark points for further phenomenological study. The points are categorized into three sets of model pairs, with a mirage mediation model and a deflected mirage mediation model in each pair. These model pairs are designed to illustrate some of the differences between pure mirage mediation models and deflected mirage mediation models, focusing on the case of large thresholds $\left(\alpha_{g}>0\right)$. The model pairs are chosen either to have similar mirage unification scales, or to illustrate the effects of a nonvanishing $\alpha_{g}$ on the 


\begin{tabular}{|l|c|c|c|c|c|c|}
\hline & Point 1 & Point 2 & Point 3 & Point 4 & Point 5 & Point 6 \\
\hline$\alpha_{m}$ & 1.9 & 1.0 & 0.6 & 0.6 & 1.0 & 1.0 \\
$\alpha_{g}$ & 0 & 0.5 & 0 & 1.0 & 0 & 0.2 \\
$M_{0}$ & 1000 & 1000 & 1000 & 1000 & 800 & 800 \\
$M_{\text {mess }}$ & NA & $10^{10}$ & NA & $10^{7}$ & NA & $10^{10}$ \\
$N$ & 0 & 3 & 0 & 3 & 0 & 3 \\
\hline$m_{\tilde{\chi}_{1}^{0}}$ & 236 & 493 & 602 & 322 & 562 & 424 \\
$m_{\tilde{\chi}_{2}^{0}}$ & 247 & 516 & 848 & 329 & 660 & 452 \\
$m_{\tilde{\chi}_{3}^{0}}$ & 936 & 698 & 1114 & 943 & 725 & 569 \\
$m_{\tilde{\chi}_{4}^{0}}$ & 954 & 718 & 1127 & 946 & 779 & 601 \\
$m_{\tilde{\chi}_{1}^{ \pm}}$ & 243 & 498 & 848 & 328 & 658 & 441 \\
$m_{\tilde{\chi}_{2}^{ \pm}}$ & 937 & 718 & 1133 & 952 & 779 & 599 \\
\hline$m_{\tilde{\tau}_{1}}$ & 676 & 700 & 763 & 717 & 594 & 556 \\
$m_{\tilde{\tau}_{2}}$ & 687 & 760 & 892 & 808 & 672 & 605 \\
$m_{\tilde{\mu}_{R}}, m_{\tilde{e}_{R}}$ & 679 & 706 & 773 & 726 & 600 & 562 \\
$m_{\tilde{\mu}_{L}}, m_{\tilde{e}_{L}}$ & 685 & 761 & 894 & 810 & 672 & 605 \\
\hline$m_{\tilde{t}_{1}}$ & 620 & 687 & 1278 & 803 & 875 & 560 \\
$m_{\tilde{t}_{2}}$ & 829 & 913 & 1579 & 1091 & 1115 & 777 \\
$m_{\tilde{b}_{1}}$ & 716 & 865 & 1542 & 1055 & 1062 & 713 \\
$m_{\tilde{b}_{2}}$ & 751 & 936 & 1624 & 1153 & 1115 & 773 \\
$m_{\tilde{c}_{R}}, m_{\tilde{u}_{R}}$ & 733 & 933 & 1639 & 1160 & 1121 & 769 \\
$m_{\tilde{c}_{L}}, m_{\tilde{u}_{L}}$ & 713 & 962 & 1695 & 1204 & 1155 & 788 \\
$m_{\tilde{s}_{R}}, m_{\tilde{d}_{R}}$ & 751 & 940 & 1633 & 1162 & 1119 & 777 \\
$m_{\tilde{s}_{L}}, m_{\tilde{d}_{L}}$ & 721 & 968 & 1702 & 1210 & 1162 & 794 \\
$m_{\tilde{g}}$ & 979 & 603 & 1816 & 431 & 1266 & 646 \\
\hline $\mathrm{LSP}_{\mathrm{SP}}$ Bino \% & $0.2 \%$ & $19.1 \%$ & $99.5 \%$ & $82.0 \%$ & $93.1 \%$ & $52.5 \%$ \\
$\mathrm{LSP}_{\mathrm{Wino}} \%$ & $0.8 \%$ & $70.0 \%$ & $0.0 \%$ & $17.0 \%$ & $0.9 \%$ & $30.1 \%$ \\
$\mathrm{LSP}_{\mathrm{P}}$ Higgsino $\%$ & $99.0 \%$ & $10.9 \%$ & $0.5 \%$ & $1.0 \%$ & $6.0 \%$ & $17.4 \%$ \\
\hline
\end{tabular}

Table 1. Input Parameters, Physical Masses, and LSP Composition for Benchmark Models. The first model in each pair is a mirage mediation model and the second is a deflected mirage mediation model with $N=3$. All masses are given in $\mathrm{GeV}$. Low energy physical masses are given at the scale $1 \mathrm{TeV}$.

renormalization group running and particle spectra. The high scale input parameters which define these points are collected in table 1 , along with the physical particle masses at the $\mathrm{TeV}$ scale and the composition of the lightest neutralino, which is the lightest superpartner (LSP) in these models. The details of the model pairs are as follows. The first model in each pair is a pure mirage mediation model with the number of vector-like $\mathbf{5}+\overline{\mathbf{5}}$ messenger fields set to $N=0$, while the second model is a deflected mirage point for which we have taken $N=3$. For all of the model points in table 1 , we have set $\tan \beta=10$ and have chosen modular weights $n_{i}=1 / 2$ for the matter fields and $n_{i}=1$ for the Higgs fields, as described previously. In each case, the deflected mirage mediation models satisfy the Higgs 

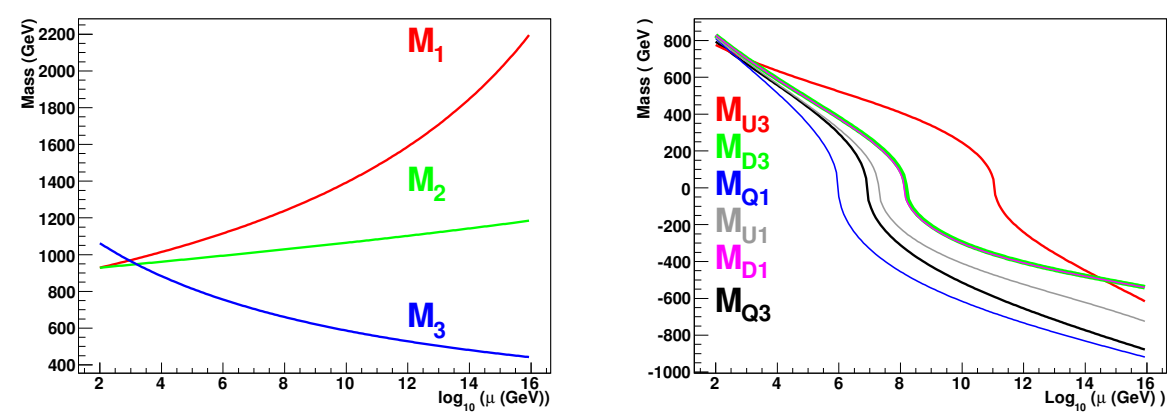

(a)
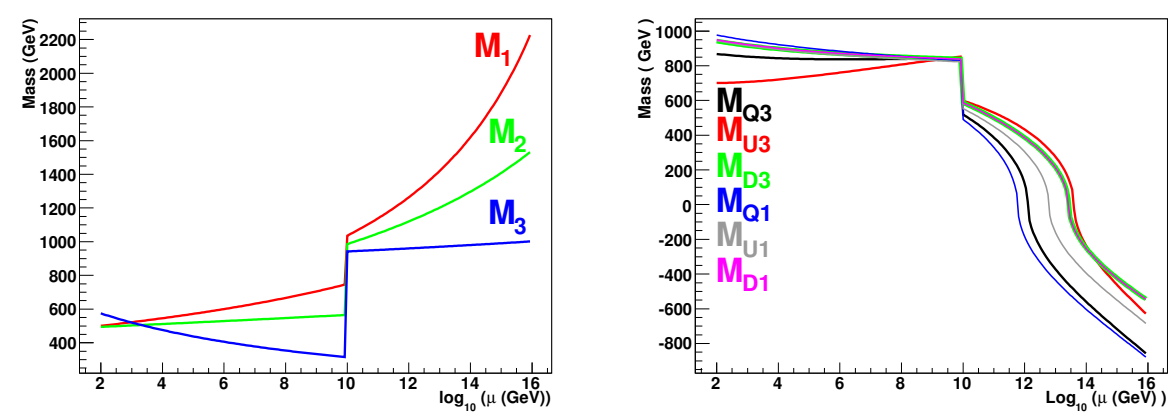

(b)

Figure 5. Benchmark Model Points 1 and 2. The renormalization group evolution of the gauginos and the scalars for (a) Point 1 and (b) Point 2. Point 1 is a mirage mediation model with $\alpha_{m}=1.9$, and Point 2 is a deflected mirage mediation scenario with $\alpha_{m}=1, \alpha_{g}=0.5$, and $M_{\text {mess }}=10^{10} \mathrm{GeV}$. Both models have $M_{0}=1 \mathrm{TeV}, N=3, \tan \beta=10$, and $\mu>0$.

bounds and the dark matter relic density constraints. The pure mirage mediation models given here do not satisfy these constraints, but are shown for sake of comparison.

The renormalization group running of the gaugino and scalar masses for the first model pair (Points 1 and 2) are given in figure 5. As can be seen from these results, the first pair are models for which there is mirage unification at the $\mathrm{TeV}$ scale in the gaugino sector. Point 1 is a pure mirage mediation model with $\alpha_{m}=1.9$ and $M_{0}=1 \mathrm{TeV}$, and hence the scalars are also unified at $\mathrm{TeV}$ energies in this case. Point 2 is a deflected mirage mediation model with $\alpha_{m}=1$ and $\alpha_{g}>0$, which has $\mathrm{TeV}$-scale gaugino mirage unification. The unification is not exact due to two-loop effects: for Point 2 the three gaugino soft masses at the electroweak scale are $M_{1}=500 \mathrm{GeV}, M_{2}=494 \mathrm{GeV}$, and $M_{3}=574 \mathrm{GeV}$, while for Point 1 they are $M_{1}=929 \mathrm{GeV}, M_{2}=929 \mathrm{GeV}$, and $M_{3}=1062 \mathrm{GeV}$. For Point 1, the electroweak symmetry breaking conditions constrain the $\mu$-parameter to a relatively small value of $\mu=239 \mathrm{GeV}$. The approximate unification at the electroweak scale is then between the gluino and the heavier pair of neutralinos and heavier chargino. The lighter pair of neutralinos are mostly Higgsino-like, as indicated by the LSP composition given in table 1. For Point 2, however, the gaugino spectrum is compressed and the gluino approximately unifies with the entire ensemble of neutral and charged gauginos. 

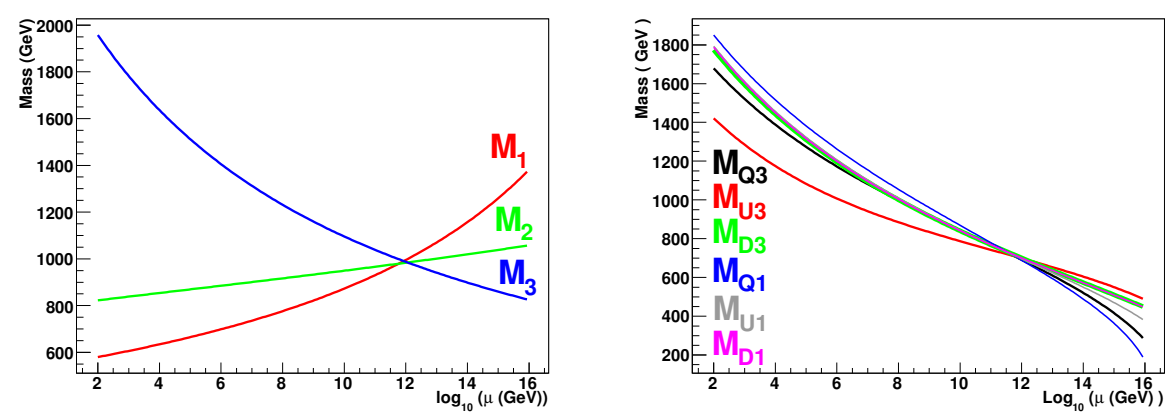

(a)
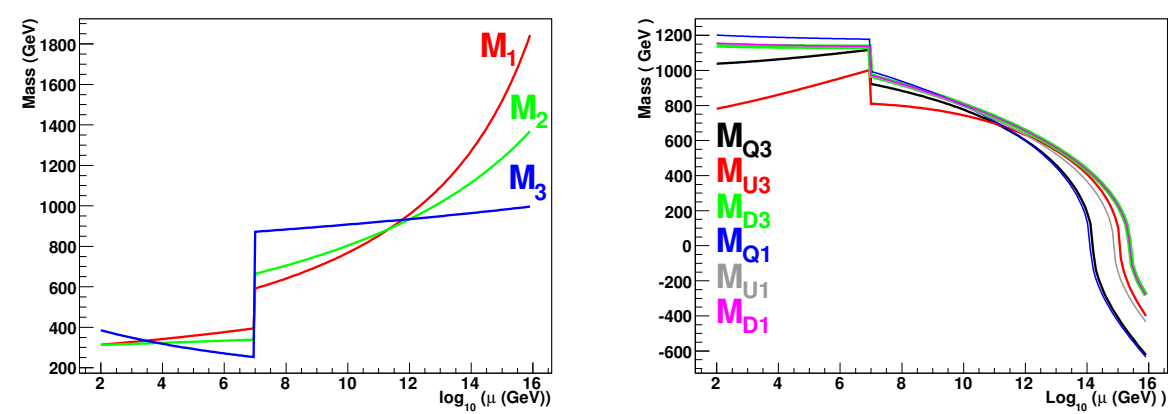

(b)

Figure 6. Benchmark Model Points 3 and 4. The renormalization group evolution of the gauginos and the scalars for (a) Point 3 and (b) Point 4. Point 3 is a mirage mediation model with $\alpha_{m}=0.6$, and Point 4 is a deflected mirage mediation scenario with $\alpha_{m}=0.6, \alpha_{g}=1$, and $M_{\text {mess }}=10^{7} \mathrm{GeV}$. Both models have $M_{0}=1 \mathrm{TeV}, N=3, \tan \beta=10$, and $\mu>0$.

The last two benchmark model pairs show the effects of keeping $\alpha_{m}$ fixed and adding nonvanishing gauge mediation contributions by including messenger fields and a nonvanishing $\alpha_{g}$. The pair represented by Points 3 and 4 is designed to show the impact of adding the effects of gauge mediation on the resulting gaugino masses. Both points have $M_{0}=1 \mathrm{TeV}$ and $\alpha_{m}=0.6$, yet the two cases have very different phenomenology. Their renormalization group evolution is shown in figure 6 . The mirage unification scale for the mirage mediation model of Point 3 is of the order of $10^{12} \mathrm{GeV}$, while the gaugino sector mirage unification scale for Point 4 is at the TeV scale due to the large threshold effects (since $\alpha_{g}=1$ ). The spectrum for the mirage mediation point is similar to typical mSUGRA/CMSSM models, with a bino-like LSP, large mass gap between the lightest and second lightest neutralinos, gluinos and squarks of roughly comparable size, and a relatively light set of sleptons. In contrast, the deflected mirage mediation point has a mixed bino/wino-like LSP, a degenerate trio of $\chi_{1}^{0}, \chi_{2}^{0}$ and $\chi_{1}^{ \pm}$, and a very light gluino relative to the squarks and sleptons. The final pair, represented by Points 5 and 6 as shown in figure 7 , are models with $M_{0}=800 \mathrm{GeV}$ which have the same values of $\alpha_{m}=1$. The deflected mirage mediation model has $\alpha_{g}=0.2$ and $M_{\text {mess }}=10^{10} \mathrm{GeV}$. The mirage mediation model (Point 5) has a mirage unification scale of order $10^{9} \mathrm{GeV}$, while the deflected mirage mediation model (Point 6) has a gaugino 

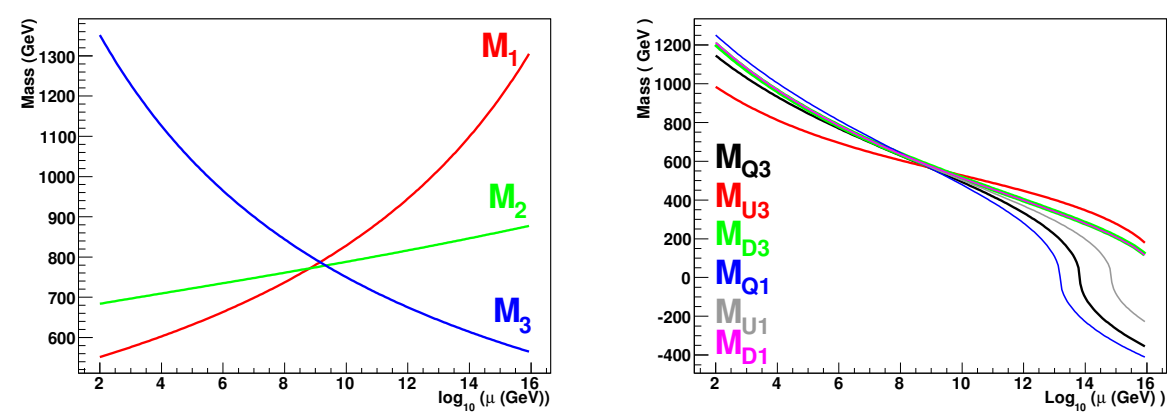

(a)
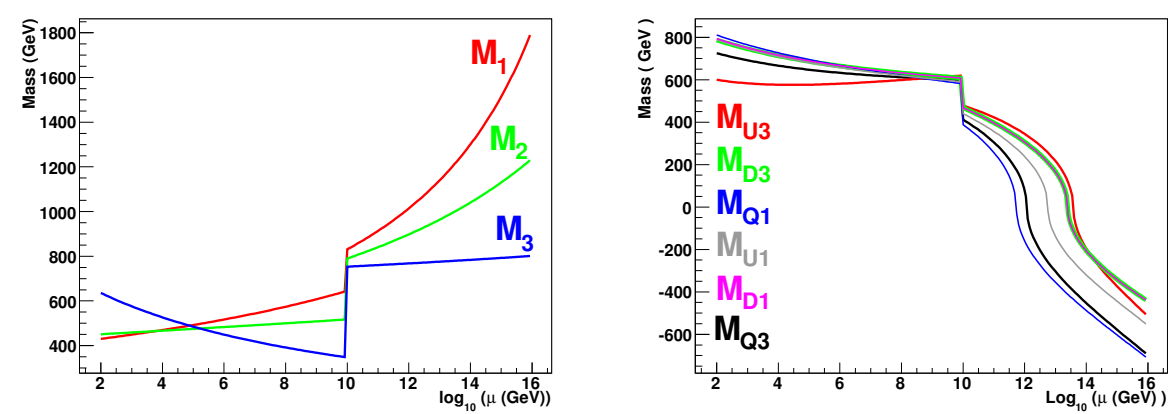

(b)

Figure 7. Benchmark Model Points 5 and 6. The renormalization group evolution of the gauginos and the scalars for (a) Point 5 and (b) Point 6 . Point 5 is a mirage mediation model with $\alpha_{m}=1$, and Point 6 is a deflected mirage mediation scenario with $\alpha_{m}=1, \alpha_{g}=0.2$, and $M_{\text {mess }}=10^{10} \mathrm{GeV}$. Both models have $M_{0}=800 \mathrm{GeV}, N=3, \tan \beta=10$, and $\mu>0$.

mirage unification scale of approximately $10^{5} \mathrm{GeV}$. These models have very similar spectra for the light superpartners, but very different values for the gluino and squark masses and the $\mu$-parameter. The mirage mediation point has a predominantly bino-like LSP, the deflected mirage mediation point has a neutralino LSP which is a mixed bino-wino-Higgsino state.

\section{Collider phenomenology}

The soft term expressions in eqs. (2.21)-(2.25) have sufficient complexity to produce a wide variety of possible low-energy superpartner spectra. The analysis of section 3 gives evidence of the wide diversity of outcomes which can arise. In this section we will turn our attention to how the addition of gauge mediation to the mirage pattern of particle masses influences the collider phenomenology of this class of models.

\subsection{Phenomenology of mirage and deflected mirage mediation model pairs}

We will begin by analyzing the collider phenomenology of the set of benchmark model pairs outlined in section 3.2. To study the collider signatures of these points at the LHC, 


\begin{tabular}{|l|c|c|c|c|c|c|}
\hline & Point 1 & Point 2 & Point 3 & Point 4 & Point 5 & Point 6 \\
\hline$\sigma_{\text {SusY }}(\mathrm{pb})$ & 5.86 & 10.86 & 0.045 & 44.71 & 0.58 & 13.26 \\
Trigger Efficiency & $84.8 \%$ & $78.9 \%$ & $99.3 \%$ & $59.4 \%$ & $98.4 \%$ & $87.1 \%$ \\
\hline
\end{tabular}

Counts per 50,000 Events

\begin{tabular}{|l|c|c|c|c|c|c|}
\hline Multijet & 5064 & 1250 & 10113 & 579 & 4645 & 1246 \\
1 Lepton & 694 & 69 & 3861 & 19 & 4266 & 445 \\
OS Dilepton & 28 & 0 & 370 & 0 & 1623 & 9 \\
SS Dilepton & 3 & 0 & 124 & 0 & 201 & 3 \\
Trilepton & 0 & 0 & 70 & 0 & 388 & 1 \\
\hline
\end{tabular}

Table 2. Gross LHC Features for Benchmark Points. The trigger efficiency is here computed using the level one trigger table of PGS4. The number of events passing our selection criteria in the multijet, single lepton plus jets, opposite-sign dilepton plus jets, same-sign dilepton plus jets, and trilepton plus jets channels are given for 50,000 generated events.

\begin{tabular}{|l|c|c|c|c|c|c|}
\hline & Point 1 & Point 2 & Point 3 & Point 4 & Point 5 & Point 6 \\
\hline Multijet & 0.17 & 0.37 & 70.30 & 0.20 & 1.90 & 0.30 \\
1 Lepton & 2.18 & 64.20 & - & 49.90 & 5.87 & 1.04 \\
OS Dilepton & 68.80 & - & - & - & 2.08 & - \\
SS Dilepton & - & - & - & - & 11.91 & - \\
Trilepton & - & - & - & - & 2.94 & - \\
\hline
\end{tabular}

Table 3. Necessary Integrated Luminosity for $5 \sigma$ Discovery in Selected Channels. The integrated luminosity (in $\mathrm{fb}^{-1}$ ) at $\sqrt{s}=14 \mathrm{TeV}$ to produce a $5 \sigma$ excess over Standard Model backgrounds is given for all cases in which $\mathcal{L}_{\text {int }} \leq 100 \mathrm{fb}^{-1}$. We require a minimum of 100 signal events in the no-lepton and single lepton channels, and a minimum of ten signal events in the multi-lepton channels.

50,000 events were generated for each model at $\sqrt{s}=14 \mathrm{TeV}$ using PYTHIA 6.4 [66]. Generated events are passed to PGS4 [67] to simulate the detector response. Events are analyzed using the PGS4 level one triggers, designed to mimic the CMS trigger tables [84]. Object-level post-trigger cuts were also imposed. We require all photons, electrons, muons and taus to have transverse momentum $p_{T} \geq 10 \mathrm{GeV}$ and $|\eta|<2.4$ and we require hadronic jets to satisfy $|\eta|<3$. Additional post-trigger level cuts were implemented for specific analyses, as described below. Our cuts are consistent (and occasionally more stringent) than those described in the SUSY search strategies section of the ATLAS [83] and CMS [84] TDRs (Technical Design Reports).

The total cross section for superpartner production is given in table 2 for each benchmark model. To a first approximation the total cross section is dependent solely on the size of the gluino mass and thus deflected mirage mediation models offer the prospect of larger LHC signals relative to comparable pure mirage mediation models. The trigger efficiency is estimated using the level one trigger table of PGS4 and represents the fraction of the 50,000 generated events that passed the trigger criteria. As we will see below, however, the actual number of events that pass post-trigger cuts will often be much smaller.

The addition of the gauge messenger sector has its most striking effect in the pair 
of Points 3 and 4, producing a difference in the total production cross section of almost three orders of magnitude. For Point 3, collecting 50,000 signal events will require over $1000 \mathrm{fb}^{-1}$ of integrated luminosity, while Point 4 achieves this in just over $1 \mathrm{fb}^{-1}$. For smaller values of $N$, the gluino mass would be larger and hence the expected signal size would diminish. Triggering efficiencies are generally slightly better for models with a less compressed gaugino mass spectrum. This results in slightly harder leptonic decay products at the final stages of cascade decays of gluinos and squarks. The PGS4 default level one trigger criteria requires leptons $\left(e^{ \pm}\right.$and $\left.\mu^{ \pm}\right)$to have $p_{T} \geq 10 \mathrm{GeV}$ in the dilepton channel, $p_{T} \geq 15 \mathrm{GeV}$ for an isolated lepton produced with a tau, and $p_{T} \geq 20 \mathrm{GeV}$ for a single isolated lepton produced in association with hard jets. In addition to this trigger requirement, standard supersymmetry search algorithms involving jets, leptons and missing transverse energy generally also demand minimum $p_{T}$ values for leptonic objects.

To demonstrate the differences between deflected mirage mediation models and their pure mirage mediation model analogs, we will here concern ourselves with counting observables associated with traditional discovery channels for supersymmetry [68], reserving a more detailed analysis of collider signatures for the following subsection. These five signatures are collected in table 2 for 50,000 generated events at $\sqrt{s}=14 \mathrm{TeV}$. These signatures are defined as follows. All five require transverse sphericity $S_{T} \geq 0.2$ and at least $250 \mathrm{GeV}$ of $\mathbb{E}_{T}$ except for the trilepton signature, where only $\mathbb{E}_{T} \geq 200 \mathrm{GeV}$ is required. Multijets here refers to events with at least four jets with the transverse momenta of the four leading jets satisfying $p_{T} \geq(200,150,50,50) \mathrm{GeV}$, respectively. For this signature we impose a veto on isolated leptons. For the single lepton, opposite-sign dilepton, same-sign dilepton and trilepton signatures we include only $e^{ \pm}$and $\mu^{ \pm}$final states and demand at least two jets with the leading jets satisfying $p_{T} \geq(100,50) \mathrm{GeV}$, respectively. The drastic reduction in the multijet and the leptonic signatures for the deflected mirage mediation models seen in table 2 is caused by the small mass gap between the LSP and either the gluino or the lightest chargino/second neutralino. This is also true of the TeV-scale mirage unification model of Point 1.

To understand how multijet signals are affected by the compressed gaugino spectrum that occurs especially at high $\alpha_{g}$ values, we have simulated our benchmark models with only gluino and squark production modes on. The results are shown in table 4 and table 5. For our benchmark models, with the notable exception of Point 3, the dominant contribution to the multijet events comes mostly from $g q \rightarrow \tilde{g} \tilde{q}_{i}$ reaction. Of this set of benchmark models, squark pair production is the next big contribution and the smallest contribution is due to gluino pair production for all cases except Point 4 (a point with a very light gluino mass), for which the situation is reversed. For Point 3, a mirage mediation point with a high value of the gluino mass, squark pair production dominates but gluino-squark production is nearly comparable, and gluino-gluino production is a small fraction.

Since the total cross sections vary significantly between the benchmark models, a more relevant number for comparison of the discovery potential between model points is the amount of integrated luminosity necessary to observe a clear excess of events over the Standard Model background. For this we generated a sample of $5 \mathrm{fb}^{-1}$ Standard Model background events, consisting of Drell-Yan, QCD dijet, $t \bar{t}, b \bar{b}, W / Z+$ jets and diboson 


\begin{tabular}{|c|ccc|ccc|ccc|}
\hline \multicolumn{3}{|c|}{$\begin{array}{c}\text { Production cross sections (pb) } \\
\text { Model }\end{array}$} & \multicolumn{3}{c|}{ Trigger Eff. (\%) } & \multicolumn{4}{c|}{ Multijet (\%) } \\
{$[\tilde{g} \tilde{g}, \tilde{q} \tilde{q}, \tilde{g} \tilde{q}]$}
\end{tabular}

Table 4. Gluino and squark production cross sections, trigger and multijet signal efficiencies.

\begin{tabular}{|c|c|ccc|}
\hline Model & \multicolumn{1}{c}{ Multijet } & $\tilde{g} \tilde{g}(\%)$ & $\tilde{q} \tilde{q}(\%)$ & $\tilde{g} \tilde{q}(\%)$ \\
\hline Point 1 & 5064 & 9.30 & 22.6 & 64.9 \\
Point 2 & 1250 & 9.44 & 35.7 & 51.8 \\
Point 3 & 10113 & 2.58 & 53.2 & 42.0 \\
Point 4 & 579 & 26.1 & 13.5 & 54.9 \\
Point 5 & 4645 & 6.85 & 34.6 & 58.8 \\
Point 6 & 1246 & 14.4 & 27.3 & 55.0 \\
\hline
\end{tabular}

Table 5. Number of multijet events per 50,000 total SUSY events and percentages of the contributions due to gluino pair production, squark pair production and gluino-squark production.

production. Scaling the weight of this sample relative to the total cross section in table 2 we can determine when a $S / \sqrt{B}=5 \sigma$ excess will be detectable at $\sqrt{s}=14 \mathrm{TeV}$. The results are given in table 3 . Note that we only extrapolate the value of $S / \sqrt{B}$ for cases where $\mathcal{L}_{\text {int }} \leq 100 \mathrm{fb}^{-1}$ and we require at least 100 signal events for the multijet and single-lepton channels and at least 10 signal events for the multi-lepton channels. With the exception of Point 3, all of these benchmark points will give clear signals in the multijet channel early in the high luminosity phase of the LHC with $\sqrt{s}=14 \mathrm{TeV}$. Leptonic discovery channels will generally take longer to observe. Point 5, despite its modest production cross-section of 0.6 picobarns, gives sizable signals in all leptonic channels within the first $10 \mathrm{fb}^{-1}$. This is largely due to the mass ordering $m_{\tilde{\chi}_{1}^{0}}<m_{\widetilde{\tau}_{1}}<m_{\tilde{\chi}_{2}^{0}}$, which does not appear in any of the deflected mirage mediation models considered here. While several points would produce $\mathcal{O}(1000)$ signal events in $1 \mathrm{fb}^{-1}$ at $\sqrt{s}=7 \mathrm{TeV}$, the reliance on multijet $+\mathbb{E}_{T}$ channels and absence of strong leptonic signals suggest that these points will be challenging to discover in the first year of LHC running.

\subsection{Influence of $\alpha_{g}$ on LHC phenomenology}

In this subsection, we wish to study in greater detail how the size of the gauge-mediated contribution to soft supersymmetry breaking affects the expected collider signatures at the LHC for deflected mirage mediation scenarios. To do so we construct four model "lines" in which the various parameters determining the soft supersymmetry breaking masses are fixed at specific values, while allowing the parameter $\alpha_{g}$ to vary. These points represent a variety of overall mass scales and spectra, many of which resemble the examples 


\begin{tabular}{|c|c|c|c|c|c|c|c|c|}
\hline \multicolumn{19}{|c|}{ Parameter Set } & $\alpha_{g}$ Value \\
\hline & $\alpha_{m}$ & $M_{0}$ & $M_{\text {mess }}$ & -1.0 & -0.5 & 0 & 0.5 & 1.0 \\
\hline Line A & 1 & $2 \mathrm{TeV}$ & $10^{12} \mathrm{GeV}$ & $\tilde{\tau}$ LSP & $\checkmark$ & $\checkmark$ & $\checkmark$ & $\checkmark \checkmark$ \\
Line B & 1 & $1 \mathrm{TeV}$ & $10^{8} \mathrm{GeV}$ & $\checkmark$ & $\checkmark \checkmark$ & $\checkmark$ & $\tilde{g}$ LSP & $\tilde{g}$ LSP \\
Line C & 0.771 & $0.8 \mathrm{TeV}$ & $10^{12} \mathrm{GeV}$ & $\checkmark$ & $\checkmark$ & $\checkmark$ & $\checkmark$ & $\checkmark$ \\
Line D & 0.755 & $0.4 \mathrm{TeV}$ & $10^{12} \mathrm{GeV}$ & $\checkmark$ & $\checkmark$ & $\checkmark$ & $\checkmark$ & $\checkmark$ \\
\hline
\end{tabular}

Table 6. Input Parameters for Benchmark Lines. For each model line we begin with the input parameter set indicated in the initial three columns. Five values of the parameter $\alpha_{g}$ were studied, keeping other parameters fixed. Points marked with a check-mark had acceptable low-energy phenomenology. Points marked with the double check-mark were studied in ref. [45].

\begin{tabular}{|c|c|c|c|c|c|c|c|c|c|c|}
\hline Model & $m_{\tilde{g}}$ & $m_{\tilde{q}_{1}}$ & $m_{\tilde{t}_{1}}$ & $m_{\mathrm{LSP}}$ & $\Delta^{0}$ & $\Delta^{ \pm}$ & $m_{\tilde{\ell}_{1}}$ & $\mathrm{~B} \%$ & $\mathrm{~W} \%$ & $\mathrm{H} \%$ \\
\hline \multicolumn{11}{|l|}{ Line $\mathrm{A}$} \\
\hline $\mathrm{A} 2$ & 2828 & 2492 & 2027 & 1400 & 175 & 179 & 1445 & $96.4 \%$ & $0.1 \%$ & $3.5 \%$ \\
\hline A3 & 2260 & 2144 & 1710 & 1265 & 132 & 132 & 1429 & $94.9 \%$ & $0.4 \%$ & $4.8 \%$ \\
\hline A 4 & 1677 & 1895 & 1479 & 1133 & 70 & 69 & 1427 & $94.1 \%$ & $1.6 \%$ & $4.3 \%$ \\
\hline A5 & 1045 & 1814 & 1380 & 977 & 30 & 1.6 & 1441 & $3.6 \%$ & $92.5 \%$ & $3.9 \%$ \\
\hline \multicolumn{11}{|l|}{ Line B } \\
\hline B1 & 1347 & 1197 & 942 & 663 & 84 & 80 & 686 & $88.7 \%$ & $1.6 \%$ & $9.6 \%$ \\
\hline B2 & 1038 & 1038 & 785 & 595 & 54 & 49 & 679 & $85.7 \%$ & $4.5 \%$ & $9.7 \%$ \\
\hline B3 & 711 & 952 & 707 & 525 & 20 & 11 & 677 & $51.4 \%$ & $37.8 \%$ & $13.4 \%$ \\
\hline \multicolumn{11}{|l|}{ Line C } \\
\hline $\mathrm{C} 1$ & 1440 & 1277 & 999 & 530 & 167 & 167 & 596 & $98.4 \%$ & $0.1 \%$ & $1.4 \%$ \\
\hline $\mathrm{C} 2$ & 1244 & 1133 & 868 & 487 & 132 & 132 & 587 & $98.0 \%$ & $0.2 \%$ & $1.8 \%$ \\
\hline C3 & 1048 & 1003 & 747 & 444 & 99 & 98 & 582 & $97.4 \%$ & $0.4 \%$ & $2.2 \%$ \\
\hline $\mathrm{C} 4$ & 847 & 894 & 647 & 402 & 66 & 65 & 580 & $96.4 \%$ & $1.0 \%$ & $2.6 \%$ \\
\hline C5 & 640 & 818 & 578 & 359 & 34 & 32 & 583 & $93.3 \%$ & $3.7 \%$ & $3.1 \%$ \\
\hline \multicolumn{11}{|l|}{ Line D } \\
\hline D1 & 752 & 672 & 496 & 254 & 75 & 73 & 297 & $94.3 \%$ & $1.1 \%$ & $4.5 \%$ \\
\hline D2 & 647 & 594 & 423 & 232 & 58 & 56 & 292 & $91.6 \%$ & $2.3 \%$ & $5.8 \%$ \\
\hline D3 & 542 & 521 & 357 & 209 & 43 & 39 & 289 & $86.3 \%$ & $5.0 \%$ & $8.7 \%$ \\
\hline D4 & 436 & 460 & 304 & 186 & 30 & 24 & 289 & $75.3 \%$ & $12.5 \%$ & $12.2 \%$ \\
\hline D5 & 325 & 415 & 273 & 161 & 22 & 12 & 290 & $51.6 \%$ & $32.6 \%$ & $15.8 \%$ \\
\hline
\end{tabular}

Table 7. Some Key Masses for Model Lines of table 4. Low-lying superpartner masses are given in units of $\mathrm{GeV}$ as well as the wavefunction composition of the LSP neutralino. Here $m_{\tilde{q}_{1}}$ is the lightest first generation squark, $m_{\tilde{t}_{1}}$ is the lighter stop, and we have defined the two mass differences $\Delta^{ \pm} \equiv m_{\tilde{\chi}_{1}^{ \pm}}-m_{\tilde{\chi}_{1}^{0}}$ and $\Delta^{0} \equiv m_{\tilde{\chi}_{2}^{0}}-m_{\tilde{\chi}_{1}^{0}}$.

shown in section 3. We summarize the relevant input parameters in table 6. For each case, we have chosen to fix $N=3, n_{i}=1 / 2$ for the matter representations and $n_{i}=1$ for the Higgs fields, and $\tan \beta=10$. Each line involves five discrete points with $\alpha_{g}=$ $\{-1.0,-0.5,0.0,0.5,1.0\}$. 
From eq. (2.21), we see that the magnitude of all three soft gaugino mass parameters will diminish as $\alpha_{g}$ is varied from $\mathcal{O}(1)$ negative values to $\mathcal{O}(1)$ positive values. The effect is strongest for the gluino, as the contribution to its mass from gauge mediation is proportional to the value of $g_{3}^{2}\left(M_{\text {mess }}\right)$ at the messenger scale. As mentioned in section 3 , we expect some value of $\alpha_{g}$ to exist above which the gluino will become the LSP, which happens for $\alpha_{g}=0.5$ and 1.0 in model line B. However, states which are charged only under $\mathrm{U}(1)_{Y}$, such as the right-handed sleptons, are largely unaffected by the variation in $\alpha_{g}$ since the threshold correction to their soft masses scales as $g_{1}^{4}\left(M_{\text {mess }}\right)$. As a result the lightest stau will have a roughly constant mass across the entire model line. For $\alpha_{g}<0$, there can be points for which the lightest neutralino is heavier than the lightest stau, which occurred for $\alpha_{g}=-1.0$ in model line A. The other points all yielded a reasonable spectrum and proper electroweak symmetry breaking at the low-energy scale.

The collider phenomenology of these models is dictated first and foremost by the overall mass scale of the superpartners - particularly those which carry SU(3) quantum numbers. The masses of these states vary dramatically with $\alpha_{g}$. The mass of the gluino and lightest stop are listed in the first two columns of table 7. Model lines $\mathrm{C}$ and $\mathrm{D}$ were chosen to produce very light squarks and gluinos. While such deflected mirage mediation models typically run into challenges with the dark matter and Higgs bounds, they were chosen here as examples for comparison because they are much more favorable with respect to the LHC than cases with heavier superpartners. Model lines A - C all involve a mass for the lightest Higgs state which satisfies $m_{h} \geq 113 \mathrm{GeV}$ over all $\alpha_{g}$ values. For line D, we have $m_{h} \leq 112 \mathrm{GeV}$ along the model line. However, we will retain this model line as the signatures are representative of a large class of deflected mirage mediation models.

As has been pointed out recently [69], once event rates are normalized to the overall mass scale of the colored superpartners the next most important factor determining the inclusive signatures for a model at the LHC is the hierarchy of low-lying superpartner masses. This is particularly true for leptonic signatures produced through the production and decay of light neutralino and chargino states. A comprehensive examination of the possible hierarchy patterns in deflected mirage mediation models in the manner of $[55,70]$ is beyond the scope of the present study and will be presented elsewhere [71]. Here we will simply list the value of the lightest neutralino mass, the lightest slepton mass (generally a scalar tau), the gluino and lightest stop mass, and the two mass differences between the lightest neutralino and the next two lightest gaugino states which we denote as

$$
\begin{aligned}
\Delta^{0} & \equiv m_{\tilde{\chi}_{2}^{0}}-m_{\tilde{\chi}_{1}^{0}}, \\
\Delta^{ \pm} & \equiv m_{\tilde{\chi}_{1}^{ \pm}}-m_{\tilde{\chi}_{1}^{0}} .
\end{aligned}
$$

These values are collected in table 7 . The mass differences $\Delta^{ \pm}$and $\Delta^{0}$ decrease monotonically with increasing $\alpha_{g}$ values because the gaugino spectrum becomes more squeezed, with the result that eventually the wino is lighter than the bino. This implies a softening of the leptonic decay products of cascade decays involving these states. Hence, one typically encounters a point at which the on-shell decays of the chargino (or second-lightest neutralino) to a slepton become kinematically forbidden - further suppressing leptonic final state signatures. The above properties are common to many models in which anomaly-mediated 


\begin{tabular}{|l|c|c|c|c|c|c|}
\hline Model Point & $\sigma_{\text {SusY }}(\mathrm{pb})$ & Trigger Eff. & Multijet & 1 Lepton & OS Dilepton & Trilepton \\
\hline Line A & $1 \times 10^{-3}$ & $98.8 \%$ & 7794 & 3846 & 687 & 213 \\
\hline A2 & $5 \times 10^{-3}$ & $99.1 \%$ & 8238 & 3741 & 360 & 105 \\
A3 & 0.02 & $98.4 \%$ & 6171 & 5976 & 823 & 252 \\
A4 & 0.21 & $73.8 \%$ & 1447 & 31 & 3 & 2 \\
A5 & 0.38 & $98.4 \%$ & 4339 & 4031 & 1486 & 447 \\
\hline Line B & 1.54 & $96.8 \%$ & 3155 & 3441 & 379 & 75 \\
\hline B1 & 5.56 & $88.0 \%$ & 2409 & 182 & 0 & 0 \\
B2 & 0.25 & $98.9 \%$ & 8798 & 3784 & 398 & 90 \\
B3 & 0.59 & $98.6 \%$ & 7932 & 3588 & 310 & 68 \\
\hline Line C & 1.45 & $98.0 \%$ & 5591 & 3718 & 499 & 102 \\
\hline C1 & 3.80 & $96.1 \%$ & 2931 & 3577 & 353 & 76 \\
C2 & 11.71 & $90.2 \%$ & 2785 & 871 & 12 & 2 \\
C3 & 12.7 & $95.9 \%$ & 2680 & 2728 & 654 & 145 \\
C4 & 27.0 & $94.0 \%$ & 2274 & 2195 & 309 & 48 \\
C5 & 61.1 & $91.0 \%$ & 1328 & 1278 & 132 & 16 \\
\hline Line D & 152.0 & $84.6 \%$ & 759 & 660 & 34 & 2 \\
\hline D1 & 459.7 & $67.2 \%$ & 365 & 109 & 4 & 1 \\
D2 &
\end{tabular}

Table 8. Gross LHC Features for Model Lines of table 4. The total cross section for production of superpartners and PGS4 level one trigger efficiency are given in the first two columns. The following four columns give the number of events in each channel per 50,000 generated events. The definitions of these signatures are modified slightly from those of section 4.1 .

supersymmetry breaking becomes important [72-77]. Indeed, as $\alpha_{g}$ is increased many properties of the gaugino sector are more and more like the anomaly mediation limit, since the moduli/gravity mediated contributions cancel with the gauge mediated contributions in this case. As a partial illustration of this trend, we give the wave-function composition of the lightest neutralino in terms of bino, wino and Higgsino percentages in the final three columns of table 7 . While cases such as model line $\mathrm{C}$ will exhibit the bino-like LSP behavior characteristic of mSUGRA/CMSSM models, others such as lines B and D will allow for a much richer set of LSP properties, including cases with a "well-tempered" neutralino [63].

To analyze the signatures of these models at the LHC, 50,000 events were generated for each of the points in table 6 at $\sqrt{s}=14 \mathrm{TeV}$ using PYTHIA 6.4. The decision to use a fixed number of events, as opposed to a certain fixed integrated luminosity, is based on the widely differing total cross-sections for supersymmetric particle production across these model lines. The total supersymmetric cross-sections range from an exceedingly small $1 \mathrm{fb}$ for Point A2 to the much larger value of $0.46 \mathrm{nb}$ for Point D5, roughly following the mass scale of the SU(3)-charged superpartners. The overall supersymmetric cross-sections 


\begin{tabular}{|c|c|c|}
\hline Model Point & $\sigma_{\text {SUSY }}(\mathrm{pb})$ & Implied $\mathcal{L}_{\text {int }}\left(\mathrm{fb}^{-1}\right)$ \\
\hline \multicolumn{3}{|l|}{ Line A } \\
\hline A2 & $1 \times 10^{-3}$ & 50000 \\
\hline A3 & $5 \times 10^{-3}$ & 10000 \\
\hline A4 & 0.02 & 2500 \\
\hline A 5 & 0.21 & 238 \\
\hline \multicolumn{3}{|l|}{ Line B } \\
\hline B1 & 0.38 & 132 \\
\hline B2 & 1.54 & 32.5 \\
\hline B3 & 5.56 & 9.0 \\
\hline \multicolumn{3}{|l|}{ Line $\mathrm{C}$} \\
\hline $\mathrm{C} 1$ & 0.25 & 200 \\
\hline $\mathrm{C} 2$ & 0.59 & 84.7 \\
\hline C3 & 1.45 & 34.5 \\
\hline $\mathrm{C} 4$ & 3.80 & 13.2 \\
\hline C5 & 11.71 & 4.27 \\
\hline \multicolumn{3}{|l|}{ Line D } \\
\hline $\mathrm{D} 1$ & 12.7 & 3.94 \\
\hline D2 & 27.0 & 1.85 \\
\hline D3 & 61.1 & 0.82 \\
\hline D4 & 152.0 & 0.33 \\
\hline D5 & 459.7 & 0.11 \\
\hline
\end{tabular}

Table 9. Implied Integrated Luminosities for Benchmark Lines. The implied integrated luminosity is the required $\mathcal{L}_{\text {int }}$ needed to produce 50,000 signal events at $\sqrt{s}=14 \mathrm{TeV}$.

and combined level one trigger efficiencies are collected in table 8. As mentioned in the previous subsection, the drop in trigger efficiencies with increasing $\alpha_{g}$ is due in part to the diminishing mass gaps between low-lying gaugino states, resulting in softer jets and leptonic decay products and fewer events entering the sample via leptonic triggers.

While the total event rate for a fixed integrated luminosity clearly distinguishes the various points in each line, we are here interested in how the introduction of a non-vanishing $\alpha_{g}$ value changes the collider phenomenology from the pure mirage mediation case with $\alpha_{g}=0$. To make meaningful statements, therefore, we will work with our fixed event rate samples. The values of $\mathcal{L}_{\text {int }}$ vary from $100 \mathrm{pb}^{-1}$ to the $50 \mathrm{ab}^{-1}$, as shown in table 9.

Our purpose here is to understand the qualitative features of certain key distributions as $\alpha_{g}$ is varied, not to address the issue of discovering low energy supersymmetry through these channels. Therefore, for simplicity and clarity of presentation, in the analysis that follows we consider the deflected mirage mediation signal only and do not include Standard Model backgrounds. In our approach, we are following similar analyses in the ATLAS Physics Report which often showed signal distributions without background samples to illustrate certain features. However, we note that the cuts we impose should, in most cases, render the backgrounds to reasonable levels because they are designed for SUSY searches, 

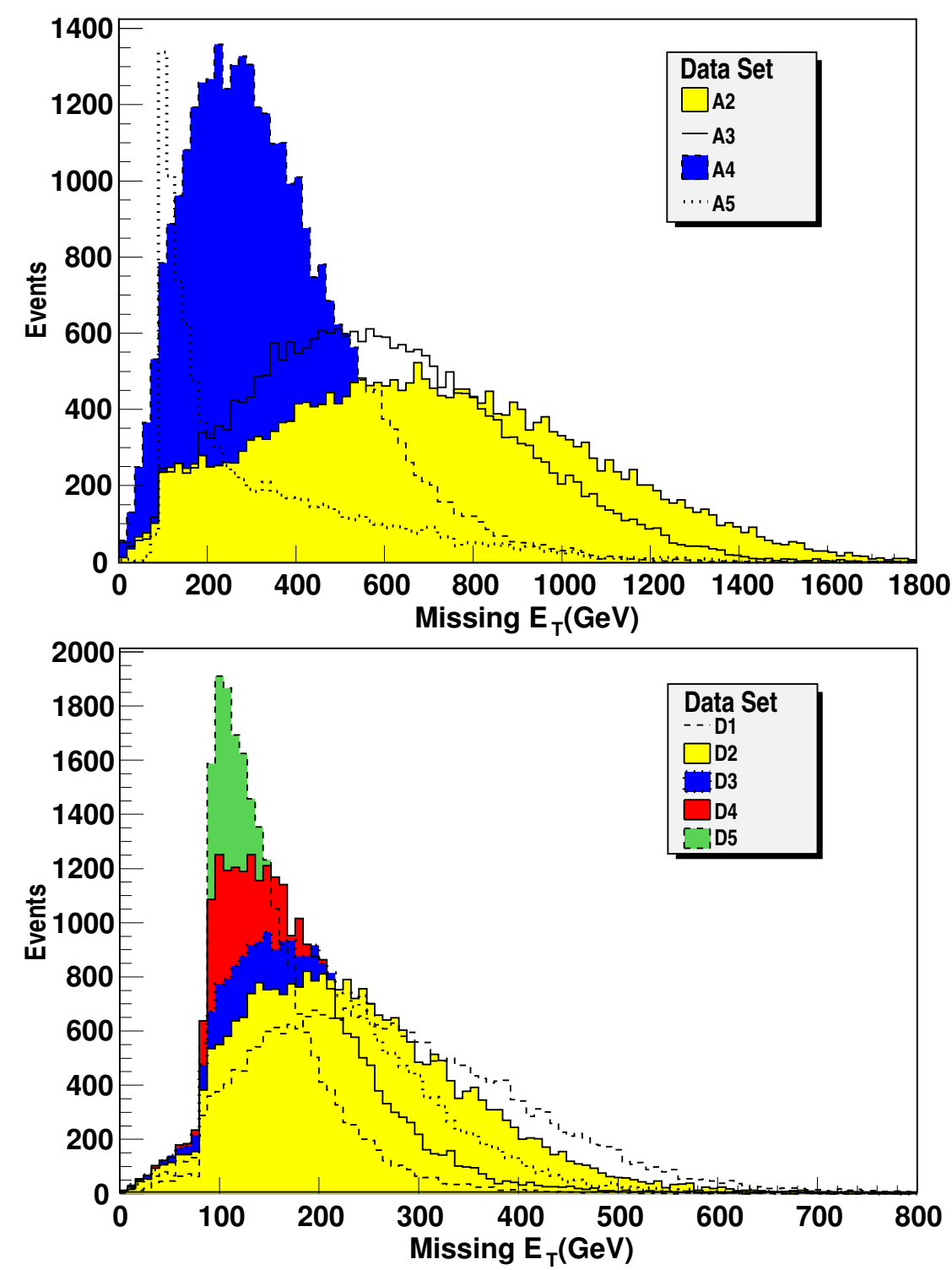

Figure 8. Missing Transverse Energy Distribution for Model Lines A and D. Upper plot is for line A while lower plot is for line D. Note that the PGS4 level one trigger menu includes a selection on events with $\not_{T} \geq 90 \mathrm{GeV}$, as indicated by the sharp change in the distributions for line D.

and in many cases are more stringent than those of ATLAS and CMS. The exceptions may be the models with compressed spectra, which may require a different cut strategy. We defer our consideration of these important issues for a future study.

In our analysis, all signature selection criteria begin with a cut on missing transverse energy of $E_{T} \geq 100 \mathrm{GeV}$ and a cut on transverse sphericity given by $S_{T} \geq 0.2$. Additional selection cuts are imposed as described below.

We begin with the inclusive multijet signature [78] which is favored as a supersymmetry discovery channel. In this subsection we will define this channel by the requirement of at 


\begin{tabular}{|c|c|c|c|c|c|c|c|c|c|}
\hline Product & \multicolumn{3}{|c|}{ Production cross sections (pb) } & \multicolumn{3}{|c|}{ Trigger Eff. (\%) } & \multicolumn{3}{|c|}{ Multijet (\%) } \\
\hline \multicolumn{10}{|l|}{ Line A } \\
\hline $\mathrm{A} 2$ & $2.98 \times 10^{-6}$ & $7.67 \times 10^{-4}$ & $1.40 \times 10^{-4}$ & 100.0 & 99.9 & 100.0 & 33.2 & 14.7 & 28.8 \\
\hline A3 & $6.39 \times 10^{-5}$ & $3.25 \times 10^{-25}$ & $1.31 \times 10^{-3}$ & 99.8 & 99.8 & 99.9 & 22.3 & 14.6 & 22.6 \\
\hline A4 & $1.73 \times 10^{-3}$ & $9.46 \times 10^{-3}$ & 0.0107 & 98.0 & 99.3 & 98.8 & 5.90 & 14.4 & 9.86 \\
\hline A5 & 0.116 & 0.0127 & 0.0774 & 53.8 & 99.7 & 98.9 & 0.792 & 17.1 & 3.88 \\
\hline \multicolumn{10}{|l|}{ Line B } \\
\hline B1 & 0.0133 & 0.198 & 0.150 & 99.2 & 98.8 & 99.2 & 15.5 & 5.13 & 12.4 \\
\hline $\mathrm{B} 2$ & 0.112 & 0.622 & 0.753 & 95.5 & 97.2 & 97.3 & 3.54 & 5.99 & 5.81 \\
\hline B3 & 1.69 & 0.848 & 2.93 & 79.2 & 94.5 & 91.2 & 1.65 & 9.20 & 4.21 \\
\hline \multicolumn{10}{|c|}{ Line $\mathrm{C}$} \\
\hline $\mathrm{C} 1$ & $7.29 \times 10^{-3}$ & 0.132 & 0.0907 & 99.7 & 99.4 & 99.7 & 25.1 & 14.1 & 23.3 \\
\hline $\mathrm{C} 2$ & 0.0261 & 0.276 & 0.246 & 99.3 & 99.0 & 99.3 & 19.4 & 12.4 & 19.4 \\
\hline C3 & 0.105 & 0.570 & 0.693 & 98.1 & 98.3 & 98.7 & 9.18 & 9.21 & 11.4 \\
\hline $\mathrm{C} 4$ & 0.499 & 1.12 & 2.03 & 94.5 & 97.1 & 97.0 & 2.55 & 6.65 & 5.30 \\
\hline C5 & 3.38 & 1.88 & 6.19 & 87.1 & 93.8 & 91.5 & 2.68 & 8.26 & 5.02 \\
\hline \multicolumn{10}{|l|}{ Line D } \\
\hline D1 & 1.16 & 4.50 & 6.31 & 97.0 & 95.9 & 97.3 & 6.99 & 2.80 & 5.75 \\
\hline D2 & 3.15 & 8.54 & 13.9 & 95.1 & 93.5 & 95.7 & 4.72 & 2.83 & 4.67 \\
\hline D3 & 9.62 & 16.6 & 32.5 & 90.9 & 89.2 & 93.2 & 1.97 & 2.08 & 2.63 \\
\hline D4 & 34.4 & 32.1 & 80.7 & 82.3 & 84.2 & 86.9 & 0.962 & 1.29 & 1.44 \\
\hline D5 & 180. & 53.9 & 218. & 61.6 & 73.4 & 70.8 & 0.598 & 1.06 & 0.918 \\
\hline
\end{tabular}

Table 10. Gluino and squark production cross sections, trigger and multijet signal efficiencies for model lines A-D.

least 4 jets, the two hardest of which satisfy $p_{T}^{\text {jet }} \geq 150 \mathrm{GeV}$ while the third and fourth hardest must satisfy $p_{T}^{\text {jet }} \geq 50 \mathrm{GeV}$. We also impose a veto on leptons $(e$ and $\mu$ ) and require $E_{T} \geq 200 \mathrm{GeV}$ for this channel. The total number of such events for each model point is listed under the heading "Multijet" in table 8. The dramatic fall in the event count for larger values of $\alpha_{g}$ is in part due to the rather severe cut on missing energy. In figure 8 we plot the distribution in $E_{T}$ across all events satisfying $S_{T} \geq 0.2$ for lines A and D. The PGS4 level one trigger requires $\mathbb{E}_{T} \geq 90 \mathrm{GeV}$ for the inclusive $\mathbb{E}_{T}$ trigger, producing a sharp drop in the observed event rate below this threshold. In the region $\alpha_{g} \gtrsim 0.5$ the distribution is clearly shifted to smaller values, with the majority of events falling below the $E_{T}=200 \mathrm{GeV}$ cut. Similar behavior occurs for the other two model lines. The supersymmetric sample size can be increased by relaxing this constraint, but only at the expense of including more of the (already sizable) Standard Model background.

In addition to being a discovery mode, the multijet channel has also been suggested as a tool for crudely measuring the overall mass scale of the superpartners. More specifically, the peak in the distribution of the variable $M_{\text {eff }}$, defined by the sum of the missing transverse 

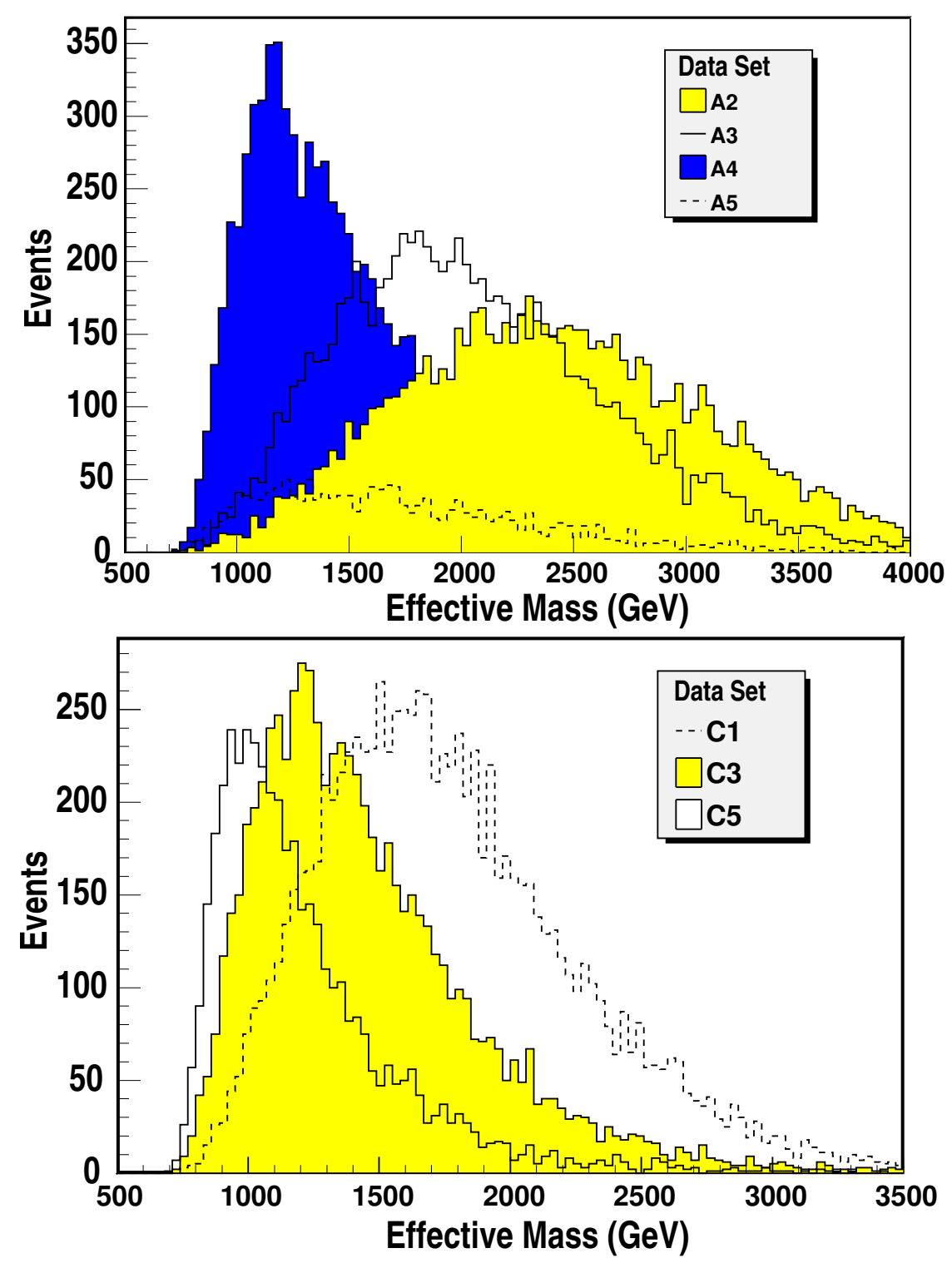

Figure 9. Effective Mass Distribution for Model Lines $A$ and $C$. The effective mass variable is here defined as $M_{\text {eff }}=E_{T}+\sum_{i=1}^{4} p_{T}^{\text {jet }}$. Smaller gluino masses are indicated by the shift in the peak of this distribution to smaller values as $\alpha_{g}$ is varied from $\alpha_{g}=-1$ to $\alpha_{g}=+1$.

energy and the transverse momenta of the four hardest jets in the event

$$
M_{\mathrm{eff}}=E_{T}+\sum_{i=1}^{4} p_{T}^{\mathrm{jet}_{\mathrm{i}}},
$$

is known to track the mass of the lowest-lying colored superpartner, most often the gluino [79]. This continues to be the case in deflected mirage mediation. In figure 9 we show the distribution in $M_{\text {eff }}$ as defined by (4.3) for line A and three values along line C. The reduction of the signal for Point A5 is directly related to the decreased missing 


\begin{tabular}{|c|c|ccc|}
\hline Model & Multijet & $\tilde{g} \tilde{g}(\%)$ & $\tilde{q} \tilde{q}(\%)$ & $\tilde{g} \tilde{q}(\%)$ \\
\hline Line A \\
\hline A2 & 7794 & 0.62 & 69.7 & 24.9 \\
A3 & 8238 & 1.74 & 57.6 & 35.9 \\
A4 & 6171 & 3.52 & 47.4 & 36.6 \\
A5 & 1447 & 8.09 & 35.4 & 48.7 \\
\hline Line B \\
\hline B1 & 4339 & 6.15 & 30.2 & 55.4 \\
B2 & 3155 & 3.93 & 37.5 & 44.1 \\
B3 & 2409 & 8.22 & 27.5 & 42.1 \\
\hline Line C & \multicolumn{5}{|l}{} \\
\hline C1 & 8798 & 4.10 & 41.7 & 47.5 \\
C2 & 7932 & 5.40 & 36.6 & 51.0 \\
C3 & 5591 & 5.83 & 31.8 & 48.0 \\
C4 & 2931 & 5.39 & 32.4 & 46.9 \\
C5 & 2785 & 12.1 & 22.4 & 43.7 \\
\hline Line D & \multicolumn{5}{l}{} \\
\hline D1 & 2680 & 11.6 & 17.7 & 51.9 \\
D2 & 2274 & 11.5 & 18.4 & 50.6 \\
D3 & 1328 & 10.6 & 19.0 & 49.2 \\
D4 & 759 & 11.9 & 15.0 & 43.7 \\
D5 & 365 & 19.7 & 12.6 & 42.2 \\
\hline
\end{tabular}

Table 11. Number of multijet events per 50,000 total SUSY events and percentages of the contributions due to gluino pair production, squark pair production and gluino-squark production.

energy in this case. For the other points in the two lines the total number of events remains roughly constant, with the peak in the distribution at a value roughly given by the gluino mass.

As in the previous subsection, to understand how multijet signals are affected as a function of $\alpha_{g}$, we have simulated our benchmark models with only gluino and squark production modes on. The results are shown in table 10 and table 11 . The number of multijet events per 50,000 SUSY events decreases with increasing $\alpha_{g}$ and as the spectrum gets lighter the production cross sections increase dramatically which actually increases the number of multijet events at constant luminosity. We normalize this huge increase in the total SUSY spectrum by fixing the number of events. Once again, the dominant contribution to the multijet events comes mostly from $g q \rightarrow \tilde{g} \tilde{q}_{i}$ reaction. Squark pair production is the next largest contribution and the smallest contribution is due to gluino pair production. As $\alpha_{g}$ value is dialed to higher values, the spectrum gets compressed but the contribution to the multijet events from gluino pair production increases for our benchmark cases. Although this increase is sometimes significant, it is not enough to make the gluino pair production the dominant mode to contribute to the multijets. 


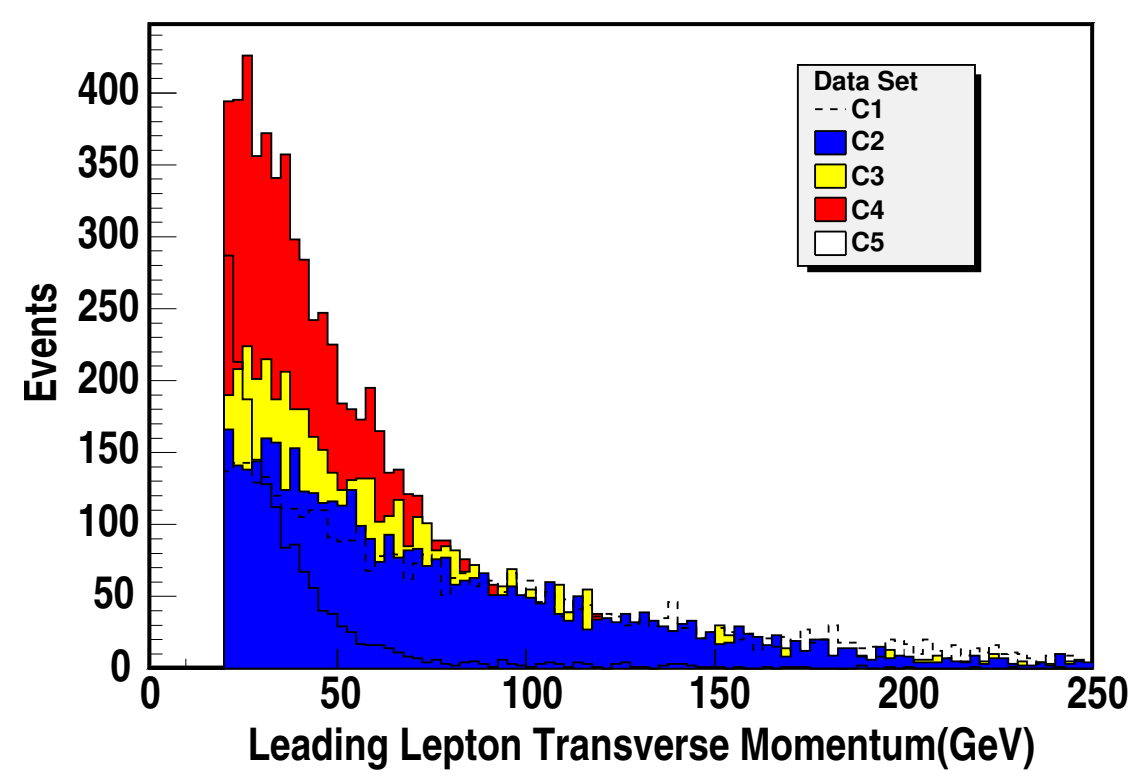

Figure 10. Transverse Momentum of the Leading Lepton for Model Line C. The transverse momentum of the lepton with the largest $p_{T}$ value is given for the five models of line C. Distributions for the other model lines are similar.

Events involving high $p_{T}$ jets and isolated leptons with missing transverse energy can also be excellent discovery modes for supersymmetry [80-82]. The final three columns of table 8 list the number of events involving one, two and three isolated leptons (respectively) satisfying $p_{T}^{\ell} \geq 20 \mathrm{GeV}$. Each of these signatures requires two or more jets satisfying $p_{T}^{\text {jet }} \geq 50 \mathrm{GeV}$ and $\mathbb{E}_{T} \geq 200 \mathrm{GeV}$. The dilepton signature involves precisely two leptons of opposite sign, though they can be of any flavor (again, lepton here implies $e$ or $\mu$ ).

Two features can be seen in the leptonic data of table 8 . The broader feature is the general reduction in leptonic activity as the value of $\alpha_{g}$ is increased along each line. The reduction is most severe for the multi-lepton signals when the mass gap between $\tilde{\chi}_{2}^{0}$ or $\tilde{\chi}_{1}^{ \pm}$ and the LSP drops below $50 \mathrm{GeV}$. For events with one or more lepton, the $p_{T}$ of the leading lepton remains relatively large. A representative example is given for the models of line $\mathrm{C}$ in figure 10. The number of events with $p_{T}^{\ell} \geq 100 \mathrm{GeV}$ remains relatively constant (hence the roughly constant number of events in the "1 Lepton" channel), while the number of events with softer leptons drops with increasing $\alpha_{g}$. The softening of the second (or third) lepton in the event is even more dramatic, resulting in fewer multi-lepton events. For models with $\Delta^{ \pm} \lesssim 5 \mathrm{GeV}$, almost all signatures of the jets + leptons variety fail to pass the trigger requirements or the leptonic $p_{T}$ cuts we impose.

The other feature involves the slight increase in multi-lepton events between Points A3 and A4 in line A and between Points $\mathrm{C} 2$ and $\mathrm{C} 3$ in line C. Both are the result of the decreasing mass differences between the lightest chargino/next-to-lightest neutralino and the LSP. For Point A3 the spoiler modes $\tilde{\chi}_{2}^{0} \rightarrow \tilde{\chi}_{1}^{0} h$ and $\tilde{\chi}_{1}^{ \pm} \rightarrow \tilde{\chi}_{1}^{0} W^{ \pm}$dominate their respective decay tables, with the former producing mostly b-jets in the final state. For Point A4, however, both of these modes are kinematically inaccessible and the three body 
decay modes $\tilde{\chi}_{2}^{0} \rightarrow \tilde{\chi}_{1}^{0} f \bar{f}$ and $\tilde{\chi}_{1}^{ \pm} \rightarrow \tilde{\chi}_{1}^{0} f f^{\prime}$ are populated. This results in a significant increase in the number of multi-lepton final states. The effect is also present in line $\mathrm{C}$, where the three body decay modes turn on only for Points C4 and C5. For Point C2 the dominant spoiler mode is $\tilde{\chi}_{2}^{0} \rightarrow \tilde{\chi}_{1}^{0} h$, but for Point C3 this mode is inaccessible and $\tilde{\chi}_{2}^{0} \rightarrow \tilde{\chi}_{1}^{0} Z$ dominates, leading to a dramatic increase in the number of opposite-sign (OS) dilepton and trilepton final states.

The opposite-sign dilepton channel is particularly important as it provides crucial information on the mass differences between low-lying gaugino eigenstates. For example, a typical strategy for measuring the mass difference between light neutralinos is to form the flavorsubtracted dilepton invariant mass for events with at least two jets satisfying $p_{T}^{\text {jet }} \geq 60 \mathrm{GeV}$, at least $200 \mathrm{GeV}$ of $E_{T}$ and two opposite-sign leptons [79]. The invariant mass distribution formed from the subset involving two leptons of opposite flavor is subtracted from that involving two of the same flavor, i.e., the combination $\left(e^{+} e^{-}+\mu^{+} \mu^{-}-e^{+} \mu^{-}-e^{-} \mu^{+}\right)$, to reduce background. For three-body decays involving $\tilde{\chi}_{2}^{0} \rightarrow \tilde{\chi}_{1}^{0} \ell \bar{\ell}$ via a virtual slepton, an edge will develop in the invariant mass distribution when $M_{\mathrm{inv}}=\Delta^{0}$, while for cascade decays involving on-shell sleptons, the edge appears at $M_{\mathrm{inv}}=\sqrt{\left(m_{\tilde{\chi}_{2}^{0}}^{2}-m_{\tilde{l}}^{2}\right)\left(m_{\tilde{l}}^{2}-m_{\tilde{\chi}_{1}^{0}}^{2}\right) / m_{\tilde{l}}^{2}}$. This distribution is plotted for lines B and D in figure 11. For both model lines, the first points (B1 and D1) are on-shell cascade decays, while the others are the three-body decays with off-shell sleptons. For line B, the third point does not yield sufficient events to produce a meaningful measurement. However, point B1 clearly shows an edge in the invariant mass as expected. Point B2, while having a much lower number of events, does indicate the expected shape based on the value of $\Delta^{0}$. For line $\mathrm{D}$ an edge in the distribution can be clearly delimited in four of the five points. For points D2-D4, the edges clearly track the decreasing mass difference between the two lightest neutralinos (4.1) as $\alpha_{g}$ is increased.

As discussed in section 3 , for large thresholds $\left(\alpha_{g}>0\right)$ the gauge mediated effects can produce a superpartner spectrum which is more compressed than the analogous case in pure mirage mediation. This can be seen in the values of the invariant mass edge in the second plot in figure 11, and can also be seen in cascade decays involving squarks and gluinos. For example, in multijet events one can construct the following ratio

$$
r_{\text {jet }} \equiv \frac{p_{T}^{\text {jet3 }}+p_{T}^{\text {jet} 4}}{p_{T}^{\text {jet1 }}+p_{T}^{\text {jet2 }}},
$$

where $p_{T}^{\text {jeti }}$ is the transverse momentum of the $i$-th hardest jet in the event. For this signature we do not impose a lepton veto, but we require that there be at least one jet with $p_{T}^{\text {jet }} \geq 100 \mathrm{GeV}$ and at least three more jets with $p_{T}^{\text {jet }} \geq 50 \mathrm{GeV}$. This signature was shown to be effective in models based on the mirage pattern of gaugino masses [85], and is here quite effective at capturing the increasing softness of the products of cascade decays as the value of $\alpha_{g}$ is changed. The distribution in the values of this ratio is plotted in figure 12 for model lines B and D. The smaller the value of $r_{\text {jet }}$, the larger the mass difference between the initially produced gluino or squark and the lighter neutralino and chargino states. As $\alpha_{g} \rightarrow 1$, the ratio tends towards $r_{\text {jet }}=0.5$ in both cases. Plots for the other model lines give similar results. Empirically we find the peak value of $r_{\text {jet }}$ tracks 


\section{Flavor-Subtracted Dilepton Invariant Mass}

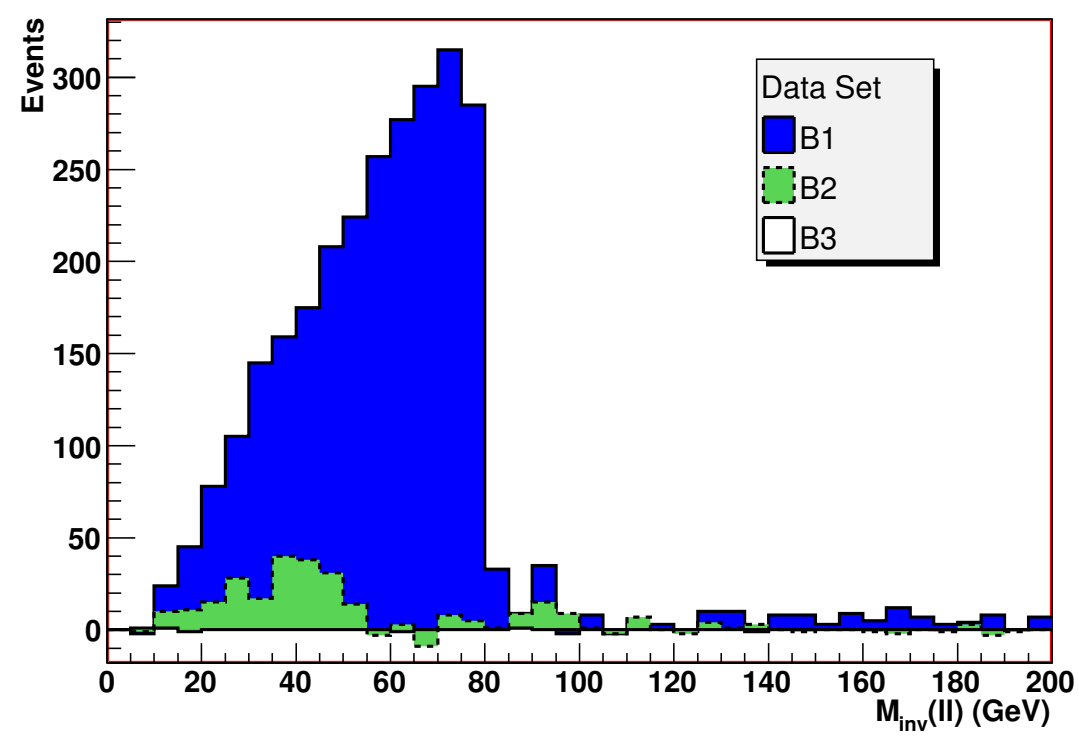

\section{Opposite-Sign Dilepton Invariant Mass}

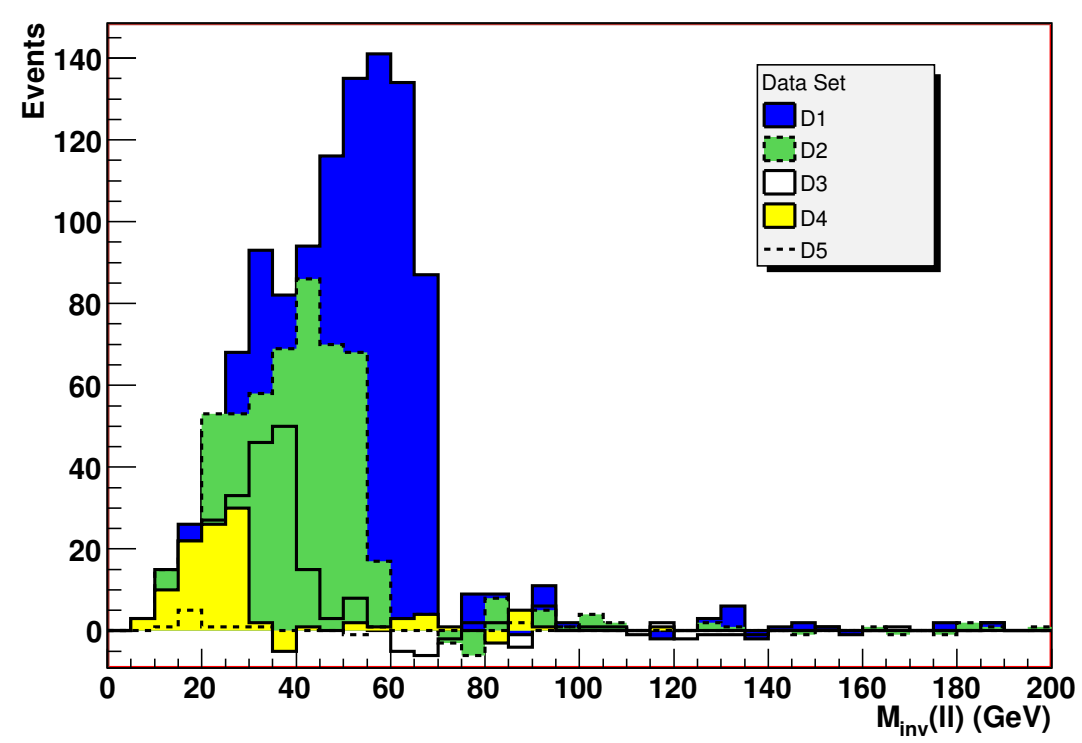

Figure 11. Flavor-Subtracted Opposite-Sign Dilepton Invariant Mass Distribution for Model Lines $B$ and $D$. The invariant mass distribution is formed from the subset involving two leptons of opposite flavor which is subtracted from that involving two of the same flavor $\left(e^{+} e^{-}+\mu^{+} \mu^{-}-\right.$ $\left.e^{+} \mu^{-}-e^{-} \mu^{+}\right)$. For enough signal events, a reasonable measurement of $\Delta^{0}$ is possible for both of these model lines.

the value of $\alpha_{g}$ with roughly the same values across each of our model lines. Signatures such as these may prove helpful at determining the value of $\alpha_{g}$ once non-universality in the gaugino sector is firmly established. 

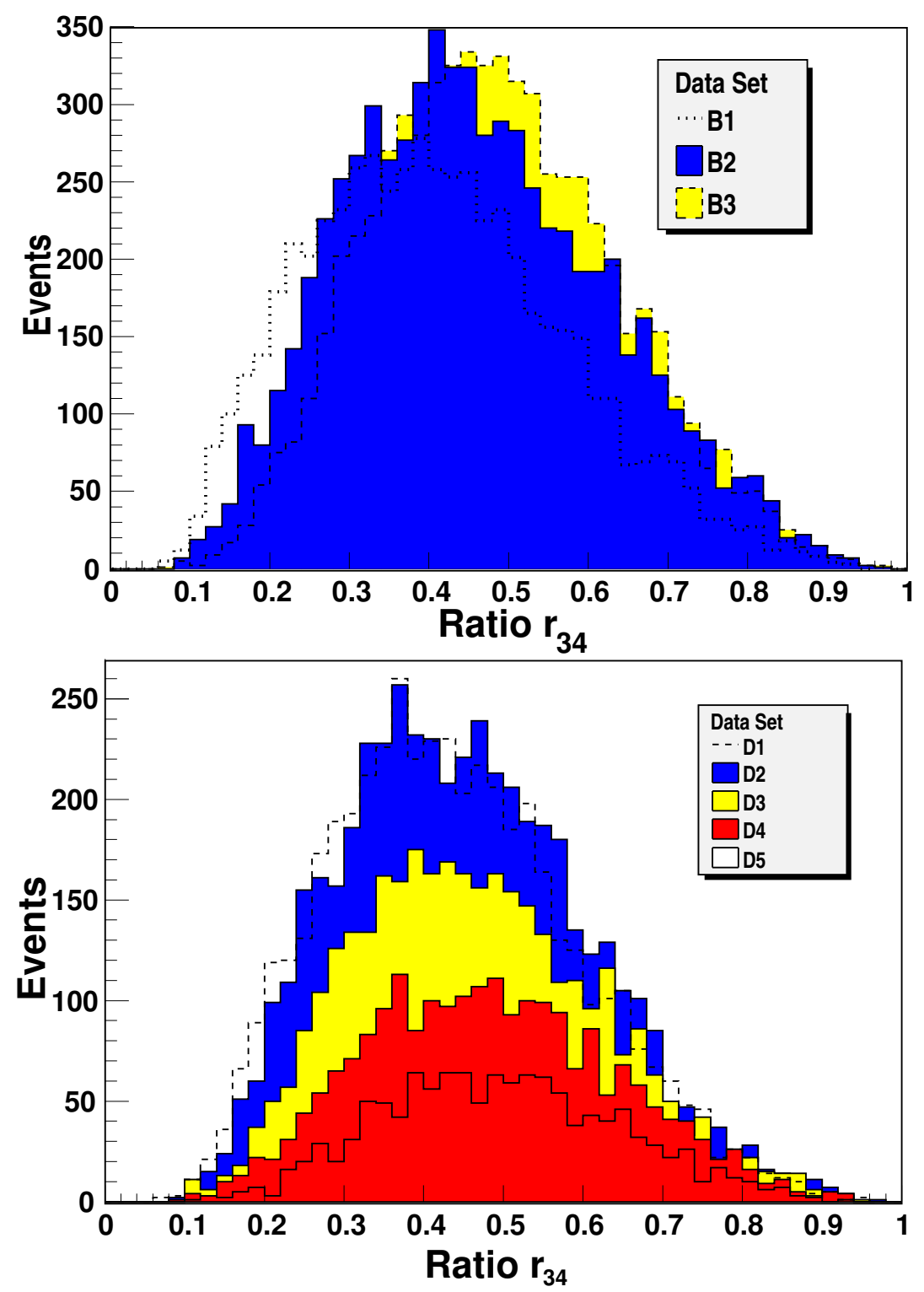

Figure 12. Distribution of Jet $p_{T}$ Ratio for Model Lines B and D. The jet $p_{T}$ ratio $r_{\text {jet }}=r_{34}$ is constructed from events with at least four jets and is defined as $r_{\mathrm{jet}}=\left(p_{T}^{\mathrm{jet} 3}+p_{T}^{\text {jet4 }}\right) /\left(p_{T}^{\text {jet1 }}+p_{T}^{\text {jet2 }}\right)$.

\section{Conclusions}

We have investigated the collider phenomenology of deflected mirage mediation, a stringmotivated "mixed" supersymmetry breaking scenario in which effects from gravity mediation, anomaly mediation, and gauge mediation all contribute to the MSSM soft terms. Our focus has been to compare the implications for LHC physics between deflected mirage mediation and the well-known mirage mediation framework, which includes gravity and anomaly mediation, but not gauge mediation. The procedure was to explore deflected mirage mediation models together with standard mirage mediation benchmark models, either 
by directly comparing models with similar gaugino mass spectra, or by investigating the effects of switching on gauge mediation starting from pure mirage mediation scenarios.

The results show that there is a broad variety of phenomenological outcomes within deflected mirage mediation, depending on the messenger scale and the size of the threshold effects from gauge mediation. One interesting class of examples have a deflected gaugino mirage unification scale at $\mathrm{TeV}$ energies, leading to a squeezed spectrum in which the gluino can be the lightest colored superpartner, which in turn results in LHC signals with softer jets and leptons than in standard MSSM models. The effects of gauge mediation can also have a large impact on the total superpartner production cross section, in some cases by several orders of magnitude. For the deflected mirage mediation examples studied here, the most robust discovery mode should be the multijet channel. The ratio of events with one lepton and high- $p_{T}$ jets to those with zero leptons should also be capable of distinguishing between the two different paradigms.

\section{Acknowledgments}

The work of B.A. and B.D.N. is supported by National Science Foundation Grant PHY0653587. L.E., I.W.K., and Y.R. are supported by the U.S. Department of Energy grant DE-FG-02-95ER40896.

\section{A Anomalous dimensions}

At one loop, the anomalous dimensions are given by

$$
\gamma_{i}=2 \sum_{a} g_{a}^{2} c_{a}\left(\Phi_{i}\right)-\frac{1}{2} \sum_{l m}\left|y_{i l m}\right|^{2}
$$

in which $c_{a}$ is the quadratic Casimir, and $y_{i l m}$ are the normalized Yukawa couplings. Here we will consider only the Yukawa couplings of the third generation $y_{t}, y_{b}$, and $y_{\tau}$. For the MSSM fields $Q, U^{c}, D^{c}, L, E^{c}, H_{u}$ and $H_{d}$, the anomalous dimensions are

$$
\begin{array}{rlrl}
\gamma_{Q, i} & =\frac{8}{3} g_{3}^{2}+\frac{3}{2} g_{2}^{2}+\frac{1}{30} g_{1}^{2}-\left(y_{t}^{2}+y_{b}^{2}\right) \delta_{i 3} \\
\gamma_{U, i} & =\frac{8}{3} g_{3}^{2}+\frac{8}{15} g_{1}^{2}-2 y_{t}^{2} \delta_{i 3}, & \gamma_{D, i} & =\frac{8}{3} g_{3}^{2}+\frac{2}{15} g_{1}^{2}-2 y_{b}^{2} \delta_{i 3}, \\
\gamma_{L, i} & =\frac{3}{2} g_{2}^{2}+\frac{3}{10} g_{1}^{2}-y_{\tau}^{2} \delta_{i 3}, & \gamma_{E, i} & =\frac{6}{5} g_{1}^{2}-2 y_{\tau}^{2} \delta_{i 3}, \\
\gamma_{H_{u}} & =\frac{3}{2} g_{2}^{2}+\frac{3}{10} g_{1}^{2}-3 y_{t}^{2}, & \gamma_{H_{d}} & =\frac{3}{2} g_{2}^{2}+\frac{3}{10} g_{1}^{2}-3 y_{b}^{2}-y_{\tau}^{2},
\end{array}
$$

respectively. Above $M_{\text {mess }}$, the beta function of the gauge couplings changes because of the messenger fields. However, $\gamma_{i}$ does not change according to eq. (A.1), and hence $\gamma_{i}^{\prime}=\gamma_{i}$. The $\dot{\gamma}_{i}$ 's are given by the expression

$$
\dot{\gamma}_{i}=2 \sum_{a} g_{a}^{4} b_{a} c_{a}\left(\Phi_{i}\right)-\sum_{l m}\left|y_{i l m}\right|^{2} b_{y_{i l m}}
$$


in which $b_{y_{i l m}}$ is the beta function for the Yukawa coupling $y_{i l m}$. The $\dot{\gamma}_{i}$ 's are given by

$$
\begin{array}{rlrl}
\dot{\gamma}_{Q, i}=\frac{8}{3} b_{3} g_{3}^{4}+\frac{3}{2} b_{2} g_{2}^{4}+\frac{1}{30} b_{1} g_{1}^{4}-\left(y_{t}^{2} b_{t}+y_{b}^{2} b_{b}\right) \delta_{i 3} \\
\dot{\gamma}_{U, i}=\frac{8}{3} b_{3} g_{3}^{4}+\frac{8}{15} b_{1} g_{1}^{4}-2 y_{t}^{2} b_{t} \delta_{i 3}, & \dot{\gamma}_{D, i}=\frac{8}{3} b_{3} g_{3}^{4}+\frac{2}{15} b_{1} g_{1}^{4}-2 y_{b}^{2} b_{b} \delta_{i 3} \\
\dot{\gamma}_{L, i}=\frac{3}{2} b_{2} g_{2}^{4}+\frac{3}{10} b_{1} g_{1}^{4}-y_{\tau}^{2} b_{\tau} \delta_{i 3}, & \dot{\gamma}_{E, i}=\frac{6}{5} b_{1} g_{1}^{4}-2 y_{\tau}^{2} b_{\tau} \delta_{i 3} \\
\dot{\gamma}_{H_{u}}=\frac{3}{2} b_{2} g_{2}^{4}+\frac{3}{10} b_{1} g_{1}^{4}-3 y_{t}^{2} b_{t}, & \dot{\gamma}_{H_{d}}=\frac{3}{2} b_{2} g_{2}^{4}+\frac{3}{10} b_{1} g_{1}^{4}-3 y_{b}^{2} b_{b}-y_{\tau}^{2} b_{\tau},
\end{array}
$$

where $b_{t}=6 y_{t}^{2}+y_{b}^{2}-\frac{16}{3} g_{3}^{2}-3 g_{2}^{2}-\frac{13}{15} g_{1}^{2}, b_{b}=y_{t}^{2}+6 y_{b}^{2}+y_{\tau}^{2}-\frac{16}{3} g_{3}^{2}-3 g_{2}^{2}-\frac{7}{15} g_{1}^{2}$ and $b_{\tau}=3 y_{b}^{2}+4 y_{\tau}^{2}-3 g_{2}^{2}-\frac{9}{5} g_{1}^{2} \cdot \dot{\gamma}_{i}^{\prime}$ is obtained by replacing $b_{a}$ with $b_{a}^{\prime}=b_{a}+N$ in eq. (A.4).

Finally, $\theta_{i}$, which appears in the mixed modulus-anomaly term in the soft scalar masssquared parameters, is given by

$$
\theta_{i}=4 \sum_{a} g_{a}^{2} c_{a}\left(Q_{i}\right)-\sum_{i, j, k}\left|y_{i j k}\right|^{2}\left(3-n_{i}-n_{j}-n_{k}\right) .
$$

For the MSSM fields, they take the form

$$
\begin{aligned}
\theta_{Q, i} & =\frac{16}{3} g_{3}^{2}+3 g_{2}^{2}+\frac{1}{15} g_{1}^{2}-2\left(y_{t}^{2}\left(3-n_{H_{u}}-n_{Q}-n_{U}\right)+y_{b}^{2}\left(3-n_{H_{d}}-n_{Q}-n_{D}\right)\right) \delta_{i 3}, \\
\theta_{U, i} & =\frac{16}{3} g_{3}^{2}+\frac{16}{15} g_{1}^{2}-4 y_{t}^{2}\left(3-n_{H_{u}}-n_{Q}-n_{U}\right) \delta_{i 3} \\
\theta_{D, i} & =\frac{16}{3} g_{3}^{2}+\frac{4}{15} g_{1}^{2}-4 y_{b}^{2}\left(3-n_{H_{d}}-n_{Q}-n_{D}\right) \delta_{i 3} \\
\theta_{L, i} & =3 g_{2}^{2}+\frac{3}{5} g_{1}^{2}-2 y_{\tau}^{2}\left(3-n_{H_{d}}-n_{L}-n_{E}\right) \delta_{i 3} \\
\theta_{E, i} & =\frac{12}{5} g_{1}^{2}-4 y_{\tau}^{2}\left(3-n_{H_{d}}-n_{L}-n_{E}\right) \delta_{i 3} \\
\theta_{H_{u}} & =3 g_{2}^{2}+\frac{3}{5} g_{1}^{2}-6 y_{t}^{2}\left(3-n_{H_{u}}-n_{Q}-n_{U}\right) \\
\theta_{H_{d}} & =3 g_{2}^{2}+\frac{3}{5} g_{1}^{2}-6 y_{b}^{2}\left(3-n_{H_{d}}-n_{Q}-n_{D}\right)-2 y_{\tau}^{2}\left(3-n_{H_{d}}-n_{L}-n_{E}\right) .
\end{aligned}
$$

As in the case of $\gamma_{i}, \theta_{i}^{\prime}$ is the same as $\theta_{i}$.

\section{References}

[1] S.P. Martin, A supersymmetry primer, hep-ph/9709356 [SPIRES].

[2] D.J.H. Chung et al., The soft supersymmetry-breaking Lagrangian: Theory and applications, Phys. Rept. 407 (2005) 1 [hep-ph/0312378] [SPIRES].

[3] A.H. Chamseddine, R.L. Arnowitt and P. Nath, Locally supersymmetric grand unification, Phys. Rev. Lett. 49 (1982) 970 [SPIRES].

[4] R. Barbieri, S. Ferrara and C.A. Savoy, Gauge models with spontaneously broken local supersymmetry, Phys. Lett. B 119 (1982) 343 [SPIRES].

[5] L.J. Hall, J.D. Lykken and S. Weinberg, Supergravity as the messenger of supersymmetry breaking, Phys. Rev. D 27 (1983) 2359 [SPIRES]. 
[6] S.K. Soni and H.A. Weldon, Analysis of the supersymmetry breaking induced by $N=1$ supergravity theories, Phys. Lett. B 126 (1983) 215 [SPIRES].

[7] H.P. Nilles, Supersymmetry, supergravity and particle physics, Phys. Rept. 110 (1984) 1 [SPIRES].

[8] A. Brignole, L.E. Ibáñez and C. Muñoz, Towards a theory of soft terms for the supersymmetric Standard Model, Nucl. Phys. B 422 (1994) 125 [Erratum ibid. B 436 (1995) 747] [hep-ph/9308271] [SPIRES].

[9] A. Brignole, L.E. Ibáñez, C. Muñoz and C. Scheich, Some issues in soft SUSY breaking terms from dilaton/moduli sectors, Z. Phys. C 74 (1997) 157 [hep-ph/9508258] [SPIRES].

[10] A. Brignole, L.E. Ibáñez and C. Muñoz, Soft supersymmetry-breaking terms from supergravity and superstring models, hep-ph/9707209 [SPIRES].

[11] L. Randall and R. Sundrum, Out of this world supersymmetry breaking, Nucl. Phys. B 557 (1999) 79 [hep-th/9810155] [SPIRES].

[12] G.F. Giudice, M.A. Luty, H. Murayama and R. Rattazzi, Gaugino mass without singlets, JHEP 12 (1998) 027 [hep-ph/9810442] [SPIRES].

[13] J.A. Bagger, T. Moroi and E. Poppitz, Anomaly mediation in supergravity theories, JHEP 04 (2000) 009 [hep-th/9911029] [SPIRES].

[14] M. Dine, W. Fischler and M. Srednicki, Supersymmetric technicolor, Nucl. Phys. B 189 (1981) 575 [SPIRES].

[15] S. Dimopoulos and S. Raby, Supercolor, Nucl. Phys. B 192 (1981) 353 [SPIRES].

[16] M. Dine and W. Fischler, A phenomenological model of particle physics based on supersymmetry, Phys. Lett. B 110 (1982) 227 [SPIRES].

[17] C.R. Nappi and B.A. Ovrut, Supersymmetric extension of the $\mathrm{SU}(3) \times \mathrm{SU}(2) \times \mathrm{U}(1)$ model, Phys. Lett. B 113 (1982) 175 [SPIRES].

[18] L. Álvarez-Gaumé, M. Claudson and M.B. Wise, Low-energy supersymmetry, Nucl. Phys. B 207 (1982) 96 [SPIRES].

[19] S. Dimopoulos and S. Raby, Geometric hierarchy, Nucl. Phys. B 219 (1983) 479 [SPIRES].

[20] M. Dine and A.E. Nelson, Dynamical supersymmetry breaking at low-energies, Phys. Rev. D 48 (1993) 1277 [hep-ph/9303230] [SPIRES].

[21] M. Dine, A.E. Nelson and Y. Shirman, Low-energy dynamical supersymmetry breaking simplified, Phys. Rev. D 51 (1995) 1362 [hep-ph/9408384] [SPIRES].

[22] M. Dine, A.E. Nelson, Y. Nir and Y. Shirman, New tools for low-energy dynamical supersymmetry breaking, Phys. Rev. D 53 (1996) 2658 [hep-ph/9507378] [SPIRES].

[23] G.F. Giudice and R. Rattazzi, Theories with gauge-mediated supersymmetry breaking, Phys. Rept. 322 (1999) 419 [hep-ph/9801271] [SPIRES].

[24] D.E. Kaplan, G.D. Kribs and M. Schmaltz, Supersymmetry breaking through transparent extra dimensions, Phys. Rev. D 62 (2000) 035010 [hep-ph/9911293] [SPIRES].

[25] Z. Chacko, M.A. Luty, A.E. Nelson and E. Ponton, Gaugino mediated supersymmetry breaking, JHEP 01 (2000) 003 [hep-ph/9911323] [SPIRES].

[26] P. Langacker, G. Paz, L.-T. Wang and I. Yavin, Z'-mediated supersymmetry breaking, Phys. Rev. Lett. 100 (2008) 041802 [arXiv:0710.1632] [SPIRES]. 
[27] P. Langacker, G. Paz, L.-T. Wang and I. Yavin, Aspects of Z'-mediated supersymmetry breaking, Phys. Rev. D 77 (2008) 085033 [arXiv:0801.3693] [SPIRES].

[28] B.C. Allanach et al., The Snowmass points and slopes: Benchmarks for SUSY searches, Eur. Phys. J. C 25 (2002) 113 [hep-ph/0202233] [SPIRES].

[29] S. Kachru, R. Kallosh, A.D. Linde and S.P. Trivedi, De Sitter vacua in string theory, Phys. Rev. D 68 (2003) 046005 [hep-th/0301240] [SPIRES].

[30] K. Choi, A. Falkowski, H.P. Nilles, M. Olechowski and S. Pokorski, Stability of flux compactifications and the pattern of supersymmetry breaking, JHEP 11 (2004) 076 [hep-th/0411066] [SPIRES].

[31] K. Choi, A. Falkowski, H.P. Nilles and M. Olechowski, Soft supersymmetry breaking in KKLT flux compactification, Nucl. Phys. B 718 (2005) 113 [hep-th/0503216] [SPIRES].

[32] K. Choi, K.S. Jeong and K.-i. Okumura, Phenomenology of mixed modulus-anomaly mediation in fluxed string compactifications and brane models, JHEP 09 (2005) 039 [hep-ph/0504037] [SPIRES].

[33] M. Endo, M. Yamaguchi and K. Yoshioka, A bottom-up approach to moduli dynamics in heavy gravitino scenario: Superpotential, soft terms and sparticle mass spectrum, Phys. Rev. D 72 (2005) 015004 [hep-ph/0504036] [SPIRES].

[34] A. Falkowski, O. Lebedev and Y. Mambrini, SUSY phenomenology of KKLT flux compactifications, JHEP 11 (2005) 034 [hep-ph/0507110] [SPIRES].

[35] H. Baer, E.-K. Park, X. Tata and T.T. Wang, Collider and dark matter searches in models with mixed modulus-anomaly mediated SUSY breaking, JHEP 08 (2006) 041 [hep-ph/0604253] [SPIRES].

[36] H. Baer, E.-K. Park, X. Tata and T.T. Wang, Collider and dark matter phenomenology of models with mirage unification, JHEP 06 (2007) 033 [hep-ph/0703024] [SPIRES].

[37] K. Choi and K.S. Jeong, Supersymmetry breaking and moduli stabilization with anomalous U(1) gauge symmetry, JHEP 08 (2006) 007 [hep-th/0605108] [SPIRES].

[38] K. Choi, K.S. Jeong and K.-I. Okumura, Flavor and CP conserving moduli mediated SUSY breaking in flux compactification, JHEP 07 (2008) 047 [arXiv:0804.4283] [SPIRES].

[39] K. Choi and H.P. Nilles, The gaugino code, JHEP 04 (2007) 006 [hep-ph/0702146] [SPIRES].

[40] K. Choi, K.S. Jeong, T. Kobayashi and K.-i. Okumura, Little SUSY hierarchy in mixed modulus-anomaly mediation, Phys. Lett. B 633 (2006) 355 [hep-ph/0508029] [SPIRES].

[41] R. Kitano and Y. Nomura, A solution to the supersymmetric fine-tuning problem within the MSSM, Phys. Lett. B 631 (2005) 58 [hep-ph/0509039] [SPIRES].

[42] O. Lebedev, H.P. Nilles and M. Ratz, A note on fine-tuning in mirage mediation, hep-ph/0511320 [SPIRES].

[43] A. Pierce and J. Thaler, Prospects for mirage mediation, JHEP 09 (2006) 017 [hep-ph/0604192] [SPIRES].

[44] K. Choi, K. Jeong, T. Kobayashi and K. Okumura, TeV scale mirage mediation and natural little SUSY hierarchy, Phys. Rev. D 75 (2007) 095012 [hep-ph/0612258] [SPIRES]. 
[45] L.L. Everett, I.-W. Kim, P. Ouyang and K.M. Zurek, Deflected mirage mediation: a framework for generalized supersymmetry breaking, Phys. Rev. Lett. 101 (2008) 101803 [arXiv:0804.0592] [SPIRES].

[46] L.L. Everett, I.-W. Kim, P. Ouyang and K.M. Zurek, Moduli stabilization and supersymmetry breaking in deflected mirage mediation, JHEP 08 (2008) 102 [arXiv:0806.2330] [SPIRES].

[47] K. Choi, K.S. Jeong, S. Nakamura, K.-I. Okumura and M. Yamaguchi, Sparticle masses in deflected mirage mediation, JHEP 04 (2009) 107 [arXiv:0901.0052] [SPIRES].

[48] M. Holmes and B.D. Nelson, Dark matter prospects in deflected mirage mediation, JCAP 07 (2009) 019 [arXiv: 0905.0674] [SPIRES].

[49] J. Alwall, M.-P. Le, M. Lisanti and J.G. Wacker, Model-independent jets plus missing energy searches, Phys. Rev. D 79 (2009) 015005 [arXiv:0809.3264] [SPIRES].

[50] J. Alwall, M.P. Le, M. Lisanti and J.G. Wacker, Searching for gluinos at the Tevatron and beyond, Int. J. Mod. Phys. A 23 (2008) 4637 [SPIRES].

[51] D. Feldman, G. Kane, R. Lu and B.D. Nelson, Dark matter as a guide toward a light gluino at the LHC, Phys. Lett. B 687 (2010) 363 [arXiv:1002.2430] [SPIRES].

[52] S.P. Martin, Exploring compressed supersymmetry with same-sign top quarks at the Large Hadron Collider, Phys. Rev. D 78 (2008) 055019 [arXiv:0807.2820] [SPIRES].

[53] S.P. Martin, The top squark-mediated annihilation scenario and direct detection of dark matter in compressed supersymmetry, Phys. Rev. D 76 (2007) 095005 [arXiv:0707.2812] [SPIRES].

[54] S.P. Martin, Compressed supersymmetry and natural neutralino dark matter from top squark-mediated annihilation to top quarks, Phys. Rev. D 75 (2007) 115005 [hep-ph/0703097] [SPIRES].

[55] C.F. Berger, J.S. Gainer, J.L. Hewett and T.G. Rizzo, Supersymmetry without prejudice, JHEP 02 (2009) 023 [arXiv: 0812.0980] [SPIRES].

[56] R. Dermisek, H.D. Kim and I.-W. Kim, Mediation of supersymmetry breaking in gauge messenger models, JHEP 10 (2006) 001 [hep-ph/0607169] [SPIRES].

[57] K.J. Bae, R. Dermisek, H.D. Kim and I.-W. Kim, Mixed bino-wino-higgsino dark matter in gauge messenger models, JCAP 08 (2007) 014 [hep-ph/0702041] [SPIRES].

[58] H. Baer, E.-K. Park, X. Tata and T.T. Wang, Collider and dark matter searches in models with mixed modulus-anomaly mediated SUSY breaking, JHEP 08 (2006) 041 [hep-ph/0604253] [SPIRES].

[59] W.S. Cho, Y.G. Kim, K.Y. Lee, C.B. Park and Y. Shimizu, LHC signature of mirage mediation, JHEP 04 (2007) 054 [hep-ph/0703163] [SPIRES].

[60] H. Baer, E.-K. Park, X. Tata and T.T. Wang, Collider and dark matter phenomenology of models with mirage unification, JHEP 06 (2007) 033 [hep-ph/0703024] [SPIRES].

[61] A. Birkedal-Hansen and B.D. Nelson, The role of Wino content in neutralino dark matter, Phys. Rev. D 64 (2001) 015008 [hep-ph/0102075] [SPIRES].

[62] A. Birkedal-Hansen and B.D. Nelson, Relic neutralino densities and detection rates with nonuniversal gaugino masses, Phys. Rev. D 67 (2003) 095006 [hep-ph/0211071] [SPIRES].

[63] N. Arkani-Hamed, A. Delgado and G.F. Giudice, The well-tempered neutralino, Nucl. Phys. B 741 (2006) 108 [hep-ph/0601041] [SPIRES]. 
[64] K. Choi, K.Y. Lee, Y. Shimizu, Y.G. Kim and K.-i. Okumura, Neutralino dark matter in mirage mediation, JCAP 12 (2006) 017 [hep-ph/0609132] [SPIRES].

[65] S.F. King and J.P. Roberts, Natural implementation of neutralino dark matter, JHEP 09 (2006) 036 [hep-ph/0603095] [SPIRES].

[66] T. Sjöstrand, S. Mrenna and P.Z. Skands, PYTHIA 6.4 Physics and Manual, JHEP 05 (2006) 026 [hep-ph/0603175] [SPIRES].

[67] http://www.physics.ucdavis.edu/c̃onway/research/software/pgs/pgs4-general.htm.

[68] ATLAS And CMS collaboration, J.G. Branson et al., High transverse momentum physics at the large hadron collider: the ATLAS and CMS collaborations,

Eur. Phys. J. direct C 4 (2002) N1 [hep-ph/0110021] [SPIRES].

[69] D. Feldman, Z. Liu and P. Nath, Sparticles at the LHC, JHEP 04 (2008) 054 [arXiv:0802.4085] [SPIRES].

[70] D. Feldman, Z. Liu and P. Nath, The landscape of sparticle mass hierarchies and their signature space at the LHC, Phys. Rev. Lett. 99 (2007) 251802 [Erratum ibid. 100 (2008) 069902] [arXiv: 0707.1873] [SPIRES].

[71] B. Altunkaynak, L. Everett, I.W. Kim, B.D. Nelson and Y. Rao, Supersymmetric particle mass hierarchies in deflected mirage mediation, in preparation.

[72] T. Gherghetta, G.F. Giudice and J.D. Wells, Phenomenological consequences of supersymmetry with anomaly-induced masses, Nucl. Phys. B 559 (1999) 27 [hep-ph/9904378] [SPIRES].

[73] J.L. Feng, T. Moroi, L. Randall, M. Strassler and S.-f. Su, Discovering supersymmetry at the Tevatron in Wino LSP scenarios, Phys. Rev. Lett. 83 (1999) 1731 [hep-ph/9904250] [SPIRES].

[74] A.J. Barr, C.G. Lester, M.A. Parker, B.C. Allanach and P. Richardson, Discovering anomaly-mediated supersymmetry at the LHC, JHEP 03 (2003) 045 [hep-ph/0208214] [SPIRES].

[75] M. Ibe, T. Moroi and T.T. Yanagida, Possible signals of Wino LSP at the Large Hadron Collider, Phys. Lett. B 644 (2007) 355 [hep-ph/0610277] [SPIRES].

[76] S. Asai, T. Moroi, K. Nishihara and T.T. Yanagida, Testing the anomaly mediation at the LHC, Phys. Lett. B 653 (2007) 81 [arXiv:0705.3086] [SPIRES].

[77] S. Asai, T. Moroi and T.T. Yanagida, Test of anomaly mediation at the LHC, Phys. Lett. B 664 (2008) 185 [arXiv:0802.3725] [SPIRES].

[78] H. Baer, C.-h. Chen, F. Paige and X. Tata, Signals for minimal supergravity at the CERN large hadron collider: Multi - jet plus missing energy channel, Phys. Rev. D 52 (1995) 2746 [hep-ph/9503271] [SPIRES].

[79] I. Hinchliffe, F.E. Paige, M.D. Shapiro, J. Soderqvist and W. Yao, Precision SUSY measurements at CERN LHC, Phys. Rev. D 55 (1997) 5520 [hep-ph/9610544] [SPIRES].

[80] H. Baer, X. Tata and J. Woodside, Multi - lepton signals from supersymmetry at hadron super colliders, Phys. Rev. D 45 (1992) 142 [SPIRES].

[81] H. Baer, C.-h. Chen, F. Paige and X. Tata, Trileptons from chargino - neutralino production at the CERN large hadron collider, Phys. Rev. D 50 (1994) 4508 [hep-ph/9404212] [SPIRES]. 
[82] H. Baer, C.h. Chen, F. Paige and X. Tata, Signals for minimal supergravity at the CERN large hadron collider II: multilepton channels, Phys. Rev. D 53 (1996) 6241 [hep-ph/9512383] [SPIRES].

[83] The ATLAS collaboration, G. Aad et al., Expected performance of the ATLAS experiment detector, trigger and physics, arXiv:0901.0512 [SPIRES].

[84] CMS collaboration, G.L. Bayatian et al., CMS technical design report, volume II: Physics performance, J. Phys. G 34 (2007) 995 [SPIRES].

[85] B. Altunkaynak, P. Grajek, M. Holmes, G. Kane and B.D. Nelson, Studying gaugino mass unification at the LHC, JHEP 04 (2009) 114 [arXiv:0901.1145] [SPIRES]. 